\title{
Optimal Information Design and Incentive Contracts with Performance Measure Manipulation*
}

\author{
Robert F. Göx ${ }^{\dagger} \quad$ Beatrice Michaeli ${ }^{\ddagger}$
}

November 18, 2019

${ }^{*}$ We thank Romana Autrey (discussant), Tim Baldenius, Snehal Banerjee, Jeremy Bertomeu, Matthew Bloomfield, Jonathan Bonham, Brian Cadman, Judson Caskey, Stefano Cascino, George Drymiotes, Eti Einhorn, Fabrizio Ferri, Slava Fos, Henry Friedman, Brandon Gipper, Ilan Guttman, Mirko Heinle, Christian Hofmann, John Hughes, Xu Jiang, Christian Laux, Roni Michaely, DJ Nanda, Lin Nan (discussant), Alexander Nezlobin, Amoray RiggsCragun, Stefan Reichelstein (discussant), Naomi Rothenberg, Georg Schneider, Doug Skinner, Austin Sudbury, Tsahi Versano, Alfred Wagenhofer, Elyashiv Wiedman, Gaoqing Zhang, and participants at the 2019 AAA meeting in San Francisco, EAA 2019 annual congress in Paphos, 11th ARW in Zurich, Summer Conference in Finance and Accounting in Jerusalem, the Tel Aviv University Conference, Junior Accounting Theory Conference, London School of Economics, University of Mannheim, and LMU Munich for helpful comments and suggestions.

${ }^{\dagger}$ University of Zurich, robert.goex@business.uzh.ch

$\ddagger$ University of California at Los Angeles, beatrice.michaeli@anderson.ucla.edu 


\section{Abstract \\ Optimal Information Design and Incentive Contracts with Performance Measure Manipulation}

We study how a firm owner motivates a manager to create value by optimally designing an information system and a compensation contract based on a manipulable performance measure. In equilibrium, the firm either implements a perfect or an uninformative system. The information system and the pay-performance sensitivity (PPS) of the compensation contract can be substitutes in a sense that the firm optimally combines a perfect information system with a low PPS or an uninformative system with a high PPS. Because the information design is endogenous, firms facing relatively high manipulation threat may offer financial incentives that are higher-powered than the ones offered by their peers facing lower manipulation threat. If the manager is in charge of implementing the information system, he chooses a perfect one unless the firm uses the information for internal control. The firm may prefer to commit to an internal control level before observing any information.

Keywords: Bayesian persuasion, ex ante information design, performance manipulation, earnings management, incentive contracting, multi-task problem 


\section{Introduction}

Because firm value is a long-term and hard to measure objective, firms frequently rely on short-term performance measures (e.g., earnings) that are prone to manipulation by managers. Compensating managers on the basis of such measures calls for low-powered incentives (Beyer, Guttman and Marinovic 2014). The reason is that strong incentives encourage manipulation (Baker 1992; Feltham and Xie 1994). However, the empirical literature provides mixed evidence regarding the association between financial incentives and earnings management and attributes it to the differences in empirical research designs and methodologies. ${ }^{1}$ How much managers manipulate depends not only on the strength of their incentives, but also on their task productivity and the degree to which their performance measures are prone to manipulation. Furthermore, the way managers react to financial incentives and, more broadly, the economic consequences of their actions, depends on the information available to them when making economic decisions. The availability of decision-relevant information is, to a large extent, determined by the type and the precision of information sources (e.g., accounting tools, business intelligence systems, or consulting services) that firms choose to acquire. Accounting for the endogenous nature of information availability, we find that, paradoxically, firms may optimally provide managers with high-powered incentives when the underlying performance measure is more prone to manipulation.

Our study predicts that firms not always benefit from acquiring sophisticated sources of information. ${ }^{2}$ The reason is that access to more precise information facilitates not only value creation but possibly also unproductive activities. Managers who understand

\footnotetext{
${ }^{1}$ Several studies show evidence consistent with a positive association between financial incentives and earnings management (e.g., Larcker, Richardson and Tuna 2007; Harris and Bromiley 2007), while others show evidence consistent with a negative association (e.g., Armstrong, Jagolinzer and Larcker 2010), or no association (e.g., Erickson, Hanlon and Maydew 2006; Baber, Kang and Liang 2007). For detailed discussion of the research methodologies see Armstrong, Jagolinzer and Larcker (2010).

${ }^{2}$ In line with this prediction, empirical studies suggest that the use of advanced information sources is not necessarily associated with a higher firm performance and varies across firms. Cagwin and Bouwman (2002) find that the use of activity-based costing is associated with a higher ROI, whereas Ittner, Lanen and Larcker (2002) find that it is only weakly related to profitability. Brynjolfsson and McElheran (2016) find that, although the use of data-driven-decision making is associated with better performance, only $30 \%$ of the manufacturing firms in US have implemented it.
} 
better how their actions translate into measures of firm performance are typically also better prepared to game the system to their own advantage and realize higher compensation or other private benefits. ${ }^{3}$ Therefore, firms face a trade-off between the additional profit derived from more informed productive decisions and the costs of more informed gaming. This trade-off influences not only the firm's choice of information sources, but also the closely related decision of how to design the compensation schemes of the firm managers. It therefore emphasizes the importance of studying information design and financial incentives simultaneously.

In this paper, we ask how firms motivate managers to create value by optimally designing the sources of decision-relevant information and compensation contracts based on manipulable performance measures. We are especially interested in how these instruments are optimally combined in solving the underlying agency problem and, more particularly, how the information design affects the pay-performance sensitivity (PPS) of the compensation contract. We address this problem in the context of an earnings management model where a firm owner or board of directors (the principal, "she") compensates a manager (the agent, "he") on the basis of short-term earnings because the terminal value is realized too late to serve as a basis for compensation. ${ }^{4}$ The earnings reflect the agent's contribution to firm value but are also affected by unproductive "window dressing" which inflates earnings but diminishes firm value.

Both activities are unobservable, personally costly to the agent, and their marginal productivities depend on an unknown parameter of the economic environment such as a market or firm characteristic that affects production or sales (e.g., customers' taste or wilngness to pay). We ask whether the principal can improve the contractual solution of the agency problem by designing an information system such as purchasing a data collection and analysis software or hiring an adviser or consultant (e.g., contracting

\footnotetext{
${ }^{3}$ Anecdotal evidence on recent fraud cases underscores the importance of the problem. A key factor in the Enron scandal was the know-how on structuring complex financial transactions and accounting procedures partly acquired through consulting services from the CPA firm Arthur Anderson (Benston and Hartgraves 2002). Likewise, Volkswagen used sophisticated engineering know-how to evade emissions standards. (Elson, Ferrere and Goosen 2015). In both cases the information used for gaming the system could have also been used to create firm value.

${ }^{4}$ The average tenure of a CEO is 6-7 years (Marcec 2018).
} 
with a market research firm to create focus groups and determine the customer's taste or willingness to pay) that provides the firm with a (potentially noisy) signal about the unknown parameter before the agent takes his actions. ${ }^{5}$ To address this question we employ a Bayesian persuasion framework where the design of the information system is chosen before the information-dependent decisions are made. ${ }^{6}$

We first show that the principal's problem of information design reduces to the choice between an uninformative and a perfect information system, and that this choice critically depends on the financial incentives of the agent. ${ }^{7}$ For a given incentive contract, information and incentives are substitutes in a sense that a higher PPS makes the choice of a perfect information system less likely. Specifically, there is a unique threshold for the PPS of the agent's contract above which the principal strictly prefers an uninformative system over a perfect one and vice versa. ${ }^{8}$

The driving force behind this result is that precise information improves the productive action of the agent and thereby the expected benefit to the principal net of the cost for which she needs to compensate the agent. However, precise information also improves the unproductive action and thereby the expected total cost due to manipulation borne by the principal. To fix ideas, suppose that the unknown parameter represents customers' taste or willingness to pay. Information about the customers' taste and willingness to pay enables the manager to predict the market demand and plan production accordingly-this increases firm value. However, knowing customers' taste and willingness to pay may also facilitate production of extra units that will be sold at a value destroying price.

\footnotetext{
${ }^{5}$ The decision for investment in database, software or hiring of experts and advisers that gather and provide managers with decision-relevant information is typically taken by a board of directors before managers have to make operation decisions.

${ }^{6}$ The order in which the principal contracts with the agent and implements the information system is irrelevant as long as those are made before the agent chooses activity levels. This is consistent with the firms' choices in practice.

${ }^{7}$ The preference for either perfect or no information is driven by the assumption that the implementation of the information system is cost-free. We discuss the implications of relaxing this assumption in Section 3.

${ }^{8}$ Any existing information within the firm, beyond the one generated by the information system, will be captured by the prior beliefs of the principal and the agent.
} 
We illustrate that, while the information effect on the principal's surplus due to production is increasing at a decreasing rate in the PPS, the information effect on the principal's surplus from manipulation is decreasing at an accelerating rate. Thus, if the PPS is sufficiently low, the overall information effect is positive and the principal benefits from implementing a system that generates perfect information. However, if the PPS is sufficiently high, the overall information effect is negative and the principal prefers an uninformative system.

Once we establish that the principal's problem simplifies to a choice between a perfect and an uninformative system for any contract, we proceed by determining the optimal PPS conditional on perfect and no information. In equilibrium, the principal chooses the pair (information system and PPS) that yields the highest value. We show that the equilibrium PPS is discontinuous and relates closely to the degree to which the performance measure is prone to gaming (i.e., manipulation treat). Specifically, in the absence of manipulation threat, the firm always benefits from implementing perfect information system because this allows the agent to take informed productive actions. Consistently, the firm also finds it optimal to provide high-powered incentives. A slight increase in manipulation threat does not discourage the firm from implementing a perfect information system but it lowers the PPS of the agent's contract. There is a critical level of manipulation threat beyond which the implementation of a perfect information system is no longer beneficial to the firm. We find that switching to an uninformative system results in an increase of the equilibrium PPS.

The preceding discussion implies that, in equilibrium, information system and PPS can be substitutes in solving the firm's incentive problem in the sense that there is always a set of firms that optimally combine a perfect information system with a low PPS and a second set of firms that optimally combine an uninformative system with a higher PPS. The former set of firms face a relatively lower manipulation threat compared with the one that the latter set of firms face. Thus, firms using performance measures that are more prone to gaming may optimally provide higher-powered incentives than firms using performance measures that are less prone to gaming. This result complements the 
predictions made by prior literature studying optimal incentives for a given information environment (e.g., Beyer, Guttman and Marinovic 2014) and may shed a new light on the mixed empirical evidence. Specifically, future empirical studies could exploit our predictions by accounting for the information environment and the manipulation threat in their research design.

We study two extensions of our model. First, we analyze the interplay between information design and internal control. Control raises the agent's manipulation cost and can be chosen by the principal before (henceforth: "early control") or after (henceforth: "late control") the signal is generated. Late control is excessive because it ignores the fact that, in equilibrium, the principal bears the agent's manipulation cost. ${ }^{9}$ Therefore, the principal prefers late control only with a perfect information system and provided that the informational benefits are higher than the cost of overinvesting in control. Second, we study the equilibrium of a slightly modified game where the agent has the authority over the information design. We first show that, in the absence of internal control, the agent always implements a perfect information system because his expected compensation is increasing in both activity levels which makes informed choices valuable for the agent. We establish the same result in the presence of early internal control. However, with late internal control, the agent chooses an uninformative system whenever the manipulation threat is sufficiently pronounced. The reason is that in this case the agent's benefit from informed actions is lower than his loss from allowing the principal to make an informed choice of control level. Moreover, in line with the qualitative predictions of our baseline setting, we identify a non-empty range of model parameters for which the PPS is monotonically increasing as the performance measure becomes more prone to gaming.

This paper contributes to the literature on performance measure manipulation. In a part of this literature the manager privately observes the firm's actual performance and issues a potentially distorted report to the principal (Arya, Glover and Sunder 1998; Dye 1988; Demski 1998). Another part of this literature is based on the multi-task version

\footnotetext{
${ }^{9}$ This result relates to Arya, Glover and Sivaramakrishnan (1997b).
} 
of the moral hazard problem (Holmström and Milgrom 1990; Feltham and Xie 1994) and considers earnings management in single-period settings (Dutta and Gigler 2002; Crocker and Slemrod 2007; Beyer, Guttman and Marinovic 2014) or in multi-period settings (Demski, Frimor and Sappington 2004; Liang 2004; Dutta and Fan 2014).

In our model the manager also inflates earnings at a personal cost but our focus is on the ability of the principal to improve the contractual solution of the agency problem by designing a system that provides decision-relevant information. In that sense, our paper is related to the literature studying the consequences of post-contract, pre-decision information for the optimal solution of a standard moral hazard model (Penno 1984; Baiman and Sivaramakrishnan 1991; Baker 1992; Bushman, Indjejikian and Penno 2000; Christensen and Feltham 2005). Different from us, these papers assume that the agent's pre-decision information is typically privately observed by the agent and not observed by the principal. Several studies (e.g., Baiman and Evans 1983; Christensen and Demski 2003; Hofmann and Rothenberg 2013) allow for the postcontract, pre-decision information to be public and discuss its desirability for solving the risk-incentive trade-off. Different from us, they focus on single-task models.

In contrast to our model, the literature on post-contract, pre-decision information typically takes the properties of the information system as given and does not consider optimal information design. We employ a Bayesian persuasion framework in which the structure of the information system is a choice of the principal. ${ }^{10}$ Georgiades and Szentes (2018) propose a continuous time agency model, where the principal can acquire informative signals to monitor the agent's effort choice. Boleslavsky and Kim (2018) study a sender-receiver game, in which the outcome distribution is jointly determined by the agent's effort and the receivers' actions. To the best of our knowledge, our paper

\footnotetext{
${ }^{10}$ Kamenica and Gentzkow (2011) formalize the idea that a sender can persuade a receiver to take a certain action by committing to an information design. Earlier studies have also considered ex ante commitment to information dissemination (Arya, Glover and Sivaramakrishnan 1997a; Göx and Wagenhofer 2009). Several studies have extended these models to settings with multiple receivers (e.g., Michaeli 2017), multiple senders (e.g., Gentzkow and Kamenica 2017), inefficient project continuation (Bertomeu and Cheynel 2015), information acquisition by receivers (Huang 2016), and the interaction between ex-ante design of public information and ex-post disclosure of private information (Friedman, Hughes and Michaeli 2019).
} 
is the first to consider such model in the context of an earnings management problem.

Our paper also relates to the signal jamming models in which a manager can issue a biased report to boost market expectations about the firm value (Stein 1989; Fischer and Verrecchia 2000; Dye and Sridhar 2004; Ewert and Wagenhofer 2005). A common assumption in this literature is that the agent's compensation contract is given. An exception is Goldman and Slezak (2006), who allow for productive effort and endogenous contracts but, unlike us, assume that the marginal productivity of the agent's production and manipulation efforts are uncorrelated and common knowledge. Lastly, our model extension in section 4 relates to the literature on internal controls (Pae and Yoo 2001; Ewert and Wagenhofer 2018; Schantl and Wagenhofer 2018).

The paper proceeds as follows. Section 2 describes the economic setting. Section 3 determines the profit maximizing combinations of information system and incentive contracts in solving the firm's multi-task problem. Section 4 extends our results by considering internal controls and authority over information design. Section 5 concludes. A summary of all model variables and the proofs are in Appendix A. Appendix B includes alternative ways of modeling information design.

\section{Economic setting}

A risk neutral manager (the agent, "he") runs a firm on behalf of a risk neutral owner (the principal, "she"). ${ }^{11}$ The agent can increase the firm's terminal value $x$ by his productive activity $a_{r}$. We assume that the terminal value is not contractible. ${ }^{12}$ However, there is a potentially biased performance measure $y$ available for contracting. Subse-

\footnotetext{
${ }^{11}$ To keep the analysis tractable and focus on the interplay between the information system and the multi-task problem, we assume that all players are risk neutral (Beyer, Guttman and Marinovic 2014).

${ }^{12}$ This assumption is common in the agency literature (Holmström and Milgrom 1990; Baker 1992; Feltham and Xie 1994) and is motivated by the fact that the terminal value is realized after the relevant contracting horizon (the average CEO tenure is 6-7 years; see Marcec 2018). Since $x$ is not contractible, the principal cannot sell the firm to the agent (as an owner, the agent would have no incentives to bias the firm's earnings). Additional reasons preventing the agent from taking this position include limited wealth or lack of liquid assets on the part of the agent, private benefits of control on the part of the contracting party such as a board of directors, or shareholders' synergies with other business activities (see also Crocker and Slemrod 2007; Beyer, Guttman and Marinovic 2014).
} 
quently, we refer to $y$ as the firm's earnings. ${ }^{13}$

The firm's earnings are affected by the agent's productive activity $a_{r}$ but also by an unproductive activity $a_{t}$ that we interpret as performance manipulation or the agent's earnings management. As in the models of Goldman and Slezak (2006) or Ewert and Wagenhofer (2005), we allow that earnings management has a real effect on firm value. ${ }^{14}$ Specifically, we assume that the firm value is reduced by $\alpha(y-x)$ if earnings exceed the fundamental value, where $\alpha \geq 0$ measures the severity of the real consequences of the agent's manipulation. If $\alpha=0$, the agent's earnings manipulation has pure accounting consequences but no impact on the firm value. In contrast, if $\alpha>0$, increasing earnings by one dollar reduces the firm value by $\alpha$ dollars.

Both the value-creating and the manipulation activities are personally costly to the agent. To avoid that cost differences are pivotal for our results, we assume that the curvature of the cost function is the same for both activities, so that identical levels of production and manipulation cost the same. Specifically, we assume that the cost $C(\cdot)$ of either activity is increasing and strictly convex with $C(0)=0$ and $C^{\prime \prime}(\cdot)=c>0$. Let $s(y)$ denote the agent's compensation as a function of realized earnings $y$. To keep the model tractable, we assume that the principal relies on an affine compensation contract $s(y)=w+v y$, where $w$ is a lump sum transfer and $v \in[0,1]$ is the incentive rate or the PPS of the agent's compensation contract.

The ex-post utility of the agent is $u=s(y)-C\left(a_{r}\right)-C\left(a_{t}\right)$ and the ex-post payoff of the principal sums up to $\pi=x-\alpha(y-x)-s(y)$. We assume that the firm's fundamental value and its earnings take the form

$$
\begin{aligned}
& x=r\left(\phi_{r}\right) \cdot a_{r}, \\
& y=r\left(\phi_{r}\right) \cdot a_{r}+t\left(\phi_{t}\right) \cdot a_{t}+\varepsilon,
\end{aligned}
$$

\footnotetext{
${ }^{13}$ Many public firms use non-GAAP measures of earnings such as EBIT, EBITDA or other forms of adjusted earnings. Since these metrics are not prepared according to generally accepted accounting principles, senior management has considerable more discretion in their preparation (Larcker and Tayan 2016; Pozen and Kothari 2017; Sherman and Young 2018).

${ }^{14}$ Examples of real earnings management include over-production (Roychowdhury 2006), R\&D cuts (Baber, Fairfield, and Haggard 1991; Rozenbaum 2017), advertising decreases (Cohen, Mashruwala, and Zach 2010) and compromises in workplace safety (Caskey and Ozel 2017). These activities diminish the firm value (Bhojraj, Hribar, Picconi, and McInnis 2009; Cohen and Zarowin 2010).
} 
and are functions of the agent's activities and their marginal productivity $i\left(\phi_{i}\right), i \in$ $\{r, t\}$. The marginal productivities depend on the realizations of two random variables, $\phi_{r}$ and $\phi_{t}$, that we define in more detail below. Furthermore, $\varepsilon$ is a noise term distributed according to some known distribution, with mean zero and unbounded support. We assume that $i\left(\phi_{i}\right)$ is an affine function of $\phi_{i}$ so that $i(0)=k_{0}>0$ and $i^{\prime}\left(\phi_{i}\right)=k_{i}>0$, for $i \in\{r, t\} .^{15}$ Here, $k_{0}$ represent the deterministic part of the productivity which, without loss of generality, we normalize to one. Furthermore, $k_{i}$ is a positive constant measuring the sensitivity to the unknown parameter $\phi_{i}$. For the majority of our results only the ratio $\kappa \equiv k_{r} / k_{t}>0$ will matter.

The parameters $\phi_{r}$ and $\phi_{t}$ capture the aggregate impact of factors (e.g., product or input market conditions, the efficiency of the firm's production process, the legal and the regulatory environment) that determine the productivity of the activities. While many of these factors are activity-specific, others are common and affect the productivity of both activities. To distinguish between the two types of factors, we assume that $\phi_{i}=\theta+\xi_{i}$, where $\xi_{i} \in[\underline{\xi}, \bar{\xi}], i \in\{r, t\}$ represents the activity-specific shocks distributed according to some known distribution with mean zero. The common parameter $\theta \in$ $\Theta=[\underline{\theta}, \bar{\theta}]$ represents all variables that affect the productivity of both activities and, to ensure that the marginal productivity is non-negative for all realizations of $\phi_{i}, i \in\{r, t\}$, we assume that $\underline{\theta} \geq 0 \geq \underline{\xi}$. For example, $\theta$ could represent unknown factors affecting the market demand such as customer taste and willingness to pay. Being able to predict the market demand facilitates production planning and thereby value creation. However, knowing customer taste and willingness to pay may also facilitate production of extra units which increases the agent's compensation but destroys firm value because the extra units are sold at a very low price.

We assume that $\theta$ is distributed according to probability distribution $f(\cdot)$ and cumulative distribution $F(\cdot)$ with mean $E[\theta]$ and variance $\operatorname{Var}(\theta)$. The principal can implement an information system (e.g., acquire a data collection and analysis software or hire an adviser) that will provide a signal $\sigma \in \Sigma$ about $\theta$, where $\Sigma \supseteq \Theta$. In our moti-

\footnotetext{
${ }^{15}$ With this structure there is always a non-trivial agency problem (i.e. $a_{t}>0$ ) regardless of the expectation about $\phi_{i}$.
} 
vating example, the implementation of information system could represent contracting with a market research firm that will create focus groups to investigate the customer taste or willingness to pay and report the results to the firm. The signal is observable by the principal and the agent. ${ }^{16}$ We rule out message games and assume that the signal is neither contractible, nor verifiable by third parties such as court. ${ }^{17}$

The information system determines the probability distribution function $g(\cdot)$ and the cumulative distribution function $G(\cdot)$ of the posterior $\theta \mid \sigma$ for a given signal realization $\sigma$. Hence the choice of the information system in our model is represented by a choice of $g(\cdot) .{ }^{18}$ Consistent beliefs imply that the condition $E_{\sigma}[G(\theta \mid \sigma)]=F(\theta)$ has to hold (Bayesian plausibility). The set of all plausible distributions include, as special cases, a perfect information system and an uninformative one. In the former case it holds that $g(\theta \mid \sigma)=0$ for any $\theta \neq \sigma$ so that the information system perfectly reveals $\theta$. In the latter case, $g(\theta \mid \sigma)=f(\theta)$ so that the agent takes his decisions on the basis of his prior beliefs about $\theta .{ }^{19}$ We assume that, whenever indifferent, the principal implements a perfect information system.

The timeline of events is presented in Figure 1. At date 1, the states $\phi_{r}$ and $\phi_{t}$ are realized but not observed by the parties. At date 2 , the principal offers a contract $s(y)$ to the agent and implements an information system with design $g(\cdot)$ to maximize her expected payoff $\Pi(\cdot) \equiv E[\pi(\cdot)]$. At date 3 , the information system generates a publicly observable signal $\sigma$ about $\theta$, the agent updates his beliefs and chooses $a_{r}$ and $a_{t}$ to maximize his expected utility conditional on the signal realization $E[u(\cdot) \mid \sigma]$. At date

\footnotetext{
${ }^{16}$ It seems reasonable that the principal observes the signal generated by the information system that she implements. However, the assumption that the principal observes $\sigma$ is irrelevant for our main results.

${ }^{17}$ This assumption rules out that the principal can condition the agent's compensation contract on the realized signal.

${ }^{18}$ To illustrate and complement the general definition of the information system, we provide two more specific examples of information systems in Appendix B. An alternative interpretation of our model is that the principal contracts with a manger who has an observable ability of learning the decision-relevant parameter $\theta$. In such case, the choice of $g(\cdot)$ represents the choice of the manager to be hired.

${ }^{19}$ In our model, the prior beliefs incorporate any additional information available within the firm beyond the one generated by the information system. Hence, an uninformative system should not be interpreted as a complete lack of information.
} 


\begin{tabular}{llll}
1 & 2 & 3 & 4 \\
\hline $\begin{array}{l}\phi_{r} \text { and } \phi_{t} \\
\text { are realized }\end{array}$ & $\begin{array}{l}\text { Principal } \\
\text { offers } s(y) \text { and } \\
\operatorname{chooses} g(\cdot)\end{array}$ & $\begin{array}{l}\sigma \text { is realized; } \\
\text { The agent } \\
\text { choses } a_{r}, a_{t}\end{array}$ & $\begin{array}{l}\text { Payoffs } \\
\text { are realized }\end{array}$
\end{tabular}

Figure 1: Timeline

4, the ex-post payoffs $v$ and $\pi$ are realized.

\section{Equilibrium analysis}

We solve the model by backward induction. We begin by deriving the agent's choices at date 3 and then state the principal's optimization problem at date 2. Next, to derive the equilibrium of the game, we proceed in three steps. In step 1 we determine the principal's information system choice for a given incentive contract. In step 2 we derive the incentive contracts for the information systems found in step 1. In step 3 we determine the profit maximizing combinations of information system and incentive contracts.

\subsection{Agent's choices at date 3}

At date 3 , for a given compensation contract, information design $g(\cdot)$ and signal realization $\sigma$, the choice of activity levels maximizes the agent's expected utility conditional on the signal realization, ${ }^{20}$

$$
a_{r}, a_{t} \in \arg \max _{a_{r}, a_{t}} E[s(y) \mid \sigma]-C\left(a_{r}\right)-C\left(a_{t}\right)
$$

\footnotetext{
${ }^{20}$ Technically, the posterior expectation upon observing $\sigma$ depends also on the properties of the information system $g(\cdot)$. We suppress it to save on notation.
} 
where $E[s(y) \mid \sigma]=w+v \cdot E[y \mid \sigma]$ and $E[y \mid \sigma]=E\left[r\left(\phi_{r}\right) \mid \sigma\right] \cdot a_{r}+E\left[t\left(\phi_{t}\right) \mid \sigma\right] \cdot a_{t}$ by (2). Given our assumptions about $i\left(\phi_{i}\right)$ and $\phi_{i}, i \in\{r, t\}$, it holds that $E\left[i\left(\phi_{i}\right) \mid \sigma\right]=E[i(\theta) \mid \sigma]$. Therefore, the optimal activity levels take the form

$$
a_{i}^{o}(v, \sigma)=\frac{v}{c} \cdot E[i(\theta) \mid \sigma] \quad i \in\{r, t\}
$$

and are increasing in the incentive weight $v$ and in the posterior expectation of the marginal productivity $i(\theta)$. Furthermore, since $E[i(\theta) \mid \sigma]=1+k_{i} E[\theta \mid \sigma]$, the parameters $k_{r}$ and $k_{t}$ determine how the agent's activities are affected by his expectations about the common parameter $\theta$. Specifically, changes of $E[\theta \mid \sigma]$ have a higher impact on the agent's production decision than on his earnings management choice if $k_{r}>k_{t}(\kappa>1)$ and vice versa if $k_{r}<k_{t}(\kappa<1)$. Consequently, we say that, for a given posterior expectation of the common parameter $\theta$, the manager is more productive in value creation than in earnings manipulation when $\kappa>1$ and vice versa when $\kappa<1$. Hence low values of $\kappa$ imply high manipulation threat, whereas high values of $\kappa$ are indicative of low manipulation threat. For future reference, date 3 expected gross outcome and performance measure are:

$$
\begin{aligned}
& x^{o}(v, \sigma)=a_{r}^{o}(v, \sigma) E[r(\theta) \mid \sigma]=\frac{v}{c}(E[r(\theta) \mid \sigma])^{2} \\
& y^{o}(v, \sigma)=x^{o}(v, \sigma)+a_{t}^{o}(v, \sigma) E[t(\theta) \mid \sigma]=\frac{v}{c}\left[(E[r(\theta) \mid \sigma])^{2}+(E[t(\theta) \mid \sigma])^{2}\right] .
\end{aligned}
$$

\subsection{Principal's choice problem at date 2}

At date 2, the principal simultaneously chooses the contract $s(y)$ and the information system $g(\cdot)$ after taking into account the agent's choices and their effect on the firm value and the performance measure at date 3 . Her optimization problem is given by:

$$
\begin{array}{ll}
\max _{g(\cdot), v, w} & E\left[x^{o}(v, \sigma)-\alpha\left(y^{o}(v, \sigma)-x^{o}(v, \sigma)\right)-s\left(y^{o}(v, \sigma)\right)\right] \\
\text { s.t. } & E\left[s\left(y^{o}(v, \sigma)\right)-\sum_{i=r, t} C\left(a_{i}^{o}(v, \sigma)\right)\right] \geq 0 \\
& E_{\sigma}[G(\theta \mid \sigma)]=F(\theta)
\end{array}
$$


where $x^{o}(v, \sigma)$ and $y^{o}(v, \sigma)$ are defined in (5) and (6). Furthermore, (8) is the agent's participation constraint and (9) is the Bayesian plausibility constraint. Since the participation constraint is binding, the principal's objective function simplifies to:

$$
\Gamma(v) \equiv E[\gamma(v, \sigma)]
$$

where

$$
\gamma(v, \sigma) \equiv x^{o}(v, \sigma)-\alpha\left(y^{o}(v, \sigma)-x^{o}(v, \sigma)\right)-\sum_{i=r, t} C\left(a_{i}^{o}(v, \sigma)\right)
$$

is the total surplus of the agency as a function of the PPS, the signal realization $\sigma$ and the information system design $g(\cdot)$.

\subsubsection{The bang-bang nature of the optimal information design}

To understand the economic forces driving the simultaneous solution to the principal's choice problem, it is instructive to first focus on the direct effect of information and study the optimal choice of the information system for a given PPS.

Proposition 1 There exists a critical value $\widehat{v} \equiv \frac{2\left(\kappa^{2}-\alpha\right)}{\kappa^{2}+1}$ such that, at date 2, for given positive $v$, the principal chooses a perfect information system if $v \leq \widehat{v}$ and an uninformative system otherwise.

The result in Proposition 1 shows that the magnitude of the incentive rate is pivotal for the principal's choice of the information system. If the PPS is relatively low so that $v \leq \widehat{v}$, the principal optimally implements a system that perfectly reveals the value of $\theta$ before the agent makes his decisions. ${ }^{21}$ In contrast, if the PPS is relatively high so that $v>\widehat{v}$, the principal prefers that the agent makes his choice based on the prior expectation about $\theta$.

\footnotetext{
${ }^{21}$ The informativeness of the system can be restricted for various reasons. If there is a limit to how much can be learnt, the solution would still be bang-bang between an uninformative system and the most informative one that can be achieved. If the principal were to bear a convex cost of information precision, the precision of the information system would be a function of $v$ and $\kappa$. Similar to our result in Proposition 1, at some sufficiently high $v$ the principal would prefer to switch to uninformative system.
} 
Our result implies that incentives and information design are substitutes in the sense that low-powered incentives increase the likelihood that the principal designs a system that provides information about the state and vice versa. To gain intuition for the bang-bang character of the optimal information system, note that, as we show in the proof of Proposition 1, the principal's objective in (10) can be presented as:

$$
\Gamma(v)=\text { const }+\frac{v}{c} \cdot \frac{\left(k_{r}^{2}+k_{t}^{2}\right)}{2}(\widehat{v}-v) \cdot \operatorname{Var}(E[\theta \mid \sigma]) .
$$

The expression in (12) shows that, for a given PPS, the principal's choice of the information system boils down to choosing the variance of the conditional expectation. The impact of $\operatorname{Var}(E[\theta \mid \sigma])$ on the principal's expected profit depends on the relative magnitude of $v$ vis a vis $\widehat{v}$. If the agent faces lower-powered incentives $(v<\widehat{v})$, the principal benefits from a high variance of the posterior expectation. On the other hand, if the agent faces high-powered incentives $(v>\widehat{v})$, the principal benefits from a low variance of the posterior expectation. Lastly, at the knife-edge case when $v=\widehat{v}$, the principal is indifferent. By the Law of total variance, $\operatorname{Var}(E[\theta \mid \sigma]) \in[0, \operatorname{Var}(\theta)]$. For an uninformative system the variance of the posterior expectation is zero. In contrast, if the system is perfectly informative, the signal $\sigma$ reflects the realization of the actual parameter $\theta$ and so the variance of the posterior expectation $E[\theta \mid \sigma]$ equals the prior variance $\operatorname{Var}(\theta)$. Taking into account our indifference assumption, the principal chooses a perfect information system if $v \leq \widehat{v}$ and an uninformative system if $v>\widehat{v}$.

To get further intuition, note that the principal's objective in (10) is essentially the expectation of the total surplus in (11). Consistent with Kamenica and Gentzkow (2011) and, more fundamentally, with Jensen's inequality, the curvature of this function determines the optimal choice of the information system. Specifically, it is easy to show that, for given positive $v$, the total surplus $\gamma(v, \sigma)$ is convex in $E[\theta \mid \sigma]$ if $v \leq \widehat{v}$ and concave in $E[\theta \mid \sigma]$ if $v \geq \widehat{v}$. Rearranging the expression for the total surplus in (11) shows that $E[r(\theta) \mid \sigma]$ and $E[t(\theta) \mid \sigma]$ not only determine the activity levels but also the net benefit of production and the total cost of manipulation:

$$
\gamma(v, \sigma)=\underbrace{a_{r}^{o}(v, \sigma) E[r(\theta) \mid \sigma]-C\left(a_{r}^{o}(v, \sigma)\right)}_{\text {net benefit of production }}-\underbrace{\alpha \cdot a_{t}^{o}(v, \sigma) E[t(\theta) \mid \sigma]-C\left(a_{t}^{o}(v, \sigma)\right)}_{\text {net cost of manipulation }} .
$$


The net benefit of production is the difference between the expected terminal value $a_{r}^{o}(v, \sigma) E[r(\theta) \mid \sigma]$ and the cost of value creation $C\left(a_{r}^{o}(v, \sigma)\right)$. Since both the agent's equilibrium activity levels in (4) and the marginal effort productivity are increasing in the posterior expectation $E[\theta \mid \sigma]$, the net benefit of production is monotonically increasing and strictly convex in $E[\theta \mid \sigma]$. By the same token, the net cost of manipulation is monotonically increasing and strictly convex in $E[\theta \mid \sigma]$. However, since it enters the total surplus with a negative sign, $\gamma(v, \sigma)$ is essentially the sum of two components that are convex and concave in $E[\theta \mid \sigma]$, respectively. Clearly, if the agent's net contribution to terminal value has a stronger impact on the curvature of the total surplus then the net cost of manipulation, $\gamma(v, \sigma)$ is convex, otherwise it is concave.

The critical value $\widehat{v}$ for the contract slope above which the total surplus becomes concave is determined by equating the second derivative of $\gamma(v, \sigma)$ with respect to $E[\theta \mid \sigma]$, to zero. The second derivative is proportional to the term

$$
\underbrace{v\left(1-\frac{v}{2}\right) k_{r}^{2}}_{\text {production effect }}-\underbrace{v\left(\alpha+\frac{v}{2}\right) k_{t}^{2}}_{\text {manipulation effect }},
$$

which represents the relative importance of the value and the manipulation cost components on the curvature of the total surplus. The first term in (13) represents the positive production effect. It is increasing at a decreasing rate in the PPS and proportional to $k_{r}^{2}$, the change of the marginal productivity with respect to an increase of the conditional expectation $E[\theta \mid \sigma]$. The second term in (13) represents the negative manipulation effect. It is decreasing in $v$ at an accelerating rate and proportional to $k_{t}^{2}$, the change of the principal's manipulation cost in response to a marginal increase of $E[\theta \mid \sigma]$. Put differently, a higher PPS flattens the convex component of the total surplus and exacerbates the concavity of the second component. As a result, if $v$ is sufficiently high, the total surplus becomes concave in the posterior. Otherwise, it remains convex. Specifically, equating the expression in (13) to zero shows that, for given $k_{r}$ and $k_{t}$, if $v<\widehat{v}$ the term (13) is strictly positive; if $v>\widehat{v}$ it is strictly negative; and in the knife-edge case of $v=\widehat{v}$ it is zero. The following result is immediate without a formal proof. 
Corollary 1 The critical value $\widehat{v}$ is increasing in $\kappa$ and decreasing in $\alpha$.

To understand the intuition behind the comparative statics, consider again the term in (13). For a given incentive rate $v$, the objective function is more likely to be convex if higher values of $E[\theta \mid \sigma]$ have a stronger impact on the marginal productivity of the agent's value creating activity (higher $k_{r}$ ) but more likely to be concave if $E[\theta \mid \sigma]$ has a stronger impact on the marginal productivity of the agent's window dressing activity (higher $k_{t}$ ). Thus, the rate $\kappa=k_{r} / k_{t}$ is a key determinant of the principal's incentives to implement sources of information about $\theta$. The higher (lower) $\kappa$, the more (less) productive is the agent in creating value relative to manipulation earnings, the lower (higher) is the manipulation threat and the more (less) is the principal inclined to design a source of perfect information about the unknown productivity parameter. Likewise, a stronger real effect on the firm's terminal cash flow (higher $\alpha$ ) render it more likely that $\gamma$ is concave in the conditional expectation of $\theta$.

Taking into account the comparative statics in Corollary 1 , and because $v \in[0,1]$, our next observations are immediate without a formal proof.

\section{Corollary 2}

(i) If $\kappa<\underline{\kappa} \equiv \sqrt{\alpha}$, then $\widehat{v}<0$ and so the principal chooses an uninformative system for any $v>0$.

(ii) If $\kappa \geq \bar{\kappa} \equiv \sqrt{1+2 \alpha}$, then $\widehat{v}>1$ and so the principal chooses a perfect information system for any $v>0$.

There are two limit cases for which the choice of the information system is independent of the PPS. If the agent is sufficiently more productive in manipulating earnings than in creating firm value, i.e., the manipulation threat is sufficiently severe (so that $\kappa<\sqrt{\alpha}$ ), the principal implements an uninformative system for any $v$ because $\widehat{v}<0 \leq v$ in this case. In contrast, if the agent is sufficiently more productive in creating firm value, i.e., the manipulation threat is sufficiently mild $(\kappa>\sqrt{1+2 \alpha} \geq 1)$, the principal implements a perfect information system for any $v$ because $\widehat{v}>1 \geq v$ in this case. 
Finally, if $\kappa \in K \equiv(\underline{\kappa}, \bar{\kappa})$, the optimal information system depends on the magnitude of $v$ and the principal chooses a perfect information system only if $v<\widehat{v}$. Intuitively, the range in which the magnitude of the optimal incentive rate is pivotal for the principal's information system choice is shifted to the right if the agent's earnings management activities have real economic consequences $(\alpha>0) .{ }^{22}$ Apart from these extreme cases, the optimal information system depends on the magnitude of the PPS.

\subsubsection{Optimal incentive rates under perfect or no information}

In this section we focus on the interesting case where $\kappa \in[\underline{\kappa}, \bar{\kappa}]$ so that the optimal information system depends in a non-trivial manner on the magnitude of $v$. By Proposition 1, for any positive PPS the principal will choose either a perfect information system or an uninformative one. If the PPS is zero then the principal will be indifferent and will choose a perfect information system as per our indifference assumption. Taking into account this bang-bang nature of the optimal information system we can simplify the principal's problem to comparing the solutions of the two mutually exclusive programs:

\section{$\mathcal{P}_{I}$ (perfect information system):}

$$
\begin{aligned}
\max _{v \in[0,1]} & \Gamma(v) \\
\text { s.t. } & v<\widehat{v}, \operatorname{Var}(E[\theta \mid \sigma])=\operatorname{Var}(\theta), \kappa \in[\underline{\kappa}, \bar{\kappa}] ;
\end{aligned}
$$

\section{$\mathcal{P}_{U}$ (Uninformative system):}

$$
\begin{aligned}
\max _{v \in(0,1]} & \Gamma(v) \\
\text { s.t. } & v \geq \widehat{v}, \operatorname{Var}(E[\theta \mid \sigma])=0, \kappa \in[\underline{\kappa}, \bar{\kappa}] .
\end{aligned}
$$

As an interim step, we first derive the unconstrained PPS for programs $\mathcal{P}_{U}$ and $\mathcal{P}_{I}$ assuming that the respective constraints are satisfied. The solutions in Lemma 1 are straightforward and presented without a formal proof.

\footnotetext{
${ }^{22}$ If earnings management only affects accounting income $(\alpha=0)$, it holds that $K=(0,1)$ so that the principal always strictly prefers an uninformative system for any $v$ only if $\kappa=0$ and a perfectly informative system whenever $\kappa>1$. In contrast, suppose that $\alpha=1$. Then, $K=(1, \sqrt{3})$ and the principal strictly prefers an uninformative system for any $v$ if $\kappa<1$ and a perfect information system for all $v$ only if $\kappa>\sqrt{3}$.
} 
Lemma 1 Under information system $j \in\{U, I\}$ the principal offers

$$
v_{j}=\frac{1-\alpha \rho_{j}^{2}}{1+\rho_{j}^{2}}<1, \quad \text { where } \rho_{U}=\frac{t(E[\theta])}{r(E[\theta])}, \quad \rho_{I}=\sqrt{\frac{E\left[t(\theta)^{2}\right]}{E\left[r(\theta)^{2}\right]}}
$$

and a fixed component $w_{j}$ that satisfies (8) as an equality. The optimal PPS $v_{j}$ is decreasing in $\alpha$ and $\rho_{j}$.

Because the performance measure $y$ is an increasing function of $a_{r}$ and $a_{t}$, the agent's activity levels are proportional to the incentive rate $v$. However, the contract slope does not determine how the agent allocates his efforts between value creation and window dressing. This choice depends on the task allocation ratio $a_{t} / a_{r}$ that captures the incongruity of the performance measure $y$ with the principal's objective $x$ (Baker 1992; Feltham and Xie 1994). ${ }^{23}$ To see how the information system affects the task allocation ratio, note that $a_{t} / a_{r}=t(E[\theta]) / r(E[\theta])$ under an uninformative system and $a_{t} / a_{r}=t(\theta) / r(\theta)$ under a perfect information system. Thus, without information about $\theta$, the agent allocates his efforts according to the expected marginal productivities and with perfect information about $\theta$, he allocates his efforts according to the true marginal productivities. In the former case, the principal can predict the agent's choice for a given PPS because the expected marginal productivities are common knowledge at the contracting date. In the latter case, however, the principal can only predict the agent's choices conditional on the realized value of $\theta$. Because the information arrives only after the contract is signed, the principal must set a PPS that solves the multi-task problem for the average agent. Depending on the severity of the manipulation threat, this difference can increase or decrease the expected agency cost caused by the multi-task problem.

The fixed component $w_{j}$ is used to transfer the expected surplus, net of the agent's reservation utility, to the principal. The optimal incentive rate $v_{j}$ balances the expected benefits from inducing more productive action against the agency cost arising from the

\footnotetext{
${ }^{23}$ Specifically, suppose that $t(\theta)=0$ so that performance manipulation is not a concern. In this case, $x=y$ and $\rho_{j}=0$ which implies that the principal's and the agent's objective are perfectly aligned and the first-best productive effort is induced by an incentive rate of $v=1$. In our model, the presence of manipulation threat renders the provision of incentive rate of one not optimal.
} 
manipulation of the performance measure. Intuitively, the equilibrium PPS is low if the real effects of earnings management are more severe (high $\alpha$ ) and if the agent is expected to be relatively more productive in manipulating earnings then in creating value (high $\left.\rho_{j}\right){ }^{24}$ To illustrate this point, it is instructive to decompose the date 2 surplus into an expected value component and a second term representing the expected agency cost

$$
\Gamma_{j}(v)=\underbrace{E\left[x^{o}(v, \sigma)-C\left(a_{r}^{o}(v, \sigma)\right)\right]}_{\text {expected firm value }}-\underbrace{\rho_{j}^{2} \cdot E\left[\alpha \cdot x^{o}(v, \sigma)+C\left(a_{r}^{o}(v, \sigma)\right)\right]}_{\text {expected agency cost }}, \quad j \in\{U, I\} .
$$

The value component in (15) measures the expected net contribution of the agent's production. The expected agency cost measures the joint loss caused by the agent's personal cost of manipulating the performance measure and the real effects associated with this manipulation as measured by the parameter $\alpha$. Both components are scaled by the square of the ratios $\rho_{j}$ indicating that the agency cost is increasing as the agent becomes relatively more productive in manipulating the firm's earnings. Substituting for the agent's activity levels from the incentive constraint, it can be shown that the principal's surplus is proportional to the expression

$$
v \cdot \underbrace{\left[\left(1-\frac{v}{2}\right)\right.}_{\text {net value effect }}-\underbrace{\left.\rho_{j}^{2} \cdot\left(\alpha+\frac{v}{2}\right)\right]}_{\text {agency cost effect }}, \quad j \in\{U, I\}
$$

Maximizing the expression in (16) yields the optimal contract in (14). Since the value effect in (16) is independent of the firm's information system, the difference in the two incentive rates is determined by the agency cost effect. Particularly, since higher values of $\rho_{j}$ increase the marginal cost of monetary incentives to the principal, the optimal PPS with perfect information must be lower than the PPS with no information if $\rho_{I}>\rho_{U}$ and vice versa.

\footnotetext{
${ }^{24} \mathrm{~A}$ positive PPS requires that $\alpha \rho_{j}^{2}<1$, otherwise the principal will find it optimal to set no incentives $(v=0)$ because the net marginal benefit from inducing productive activity is lower than the marginal cost of performance manipulation. To avoid unnecessary case distinctions, we only consider model parameters where this condition is met for all information systems. That is, it holds that $\alpha \rho_{j}^{2}<1$ for $j \in\{U, I\}$.
} 


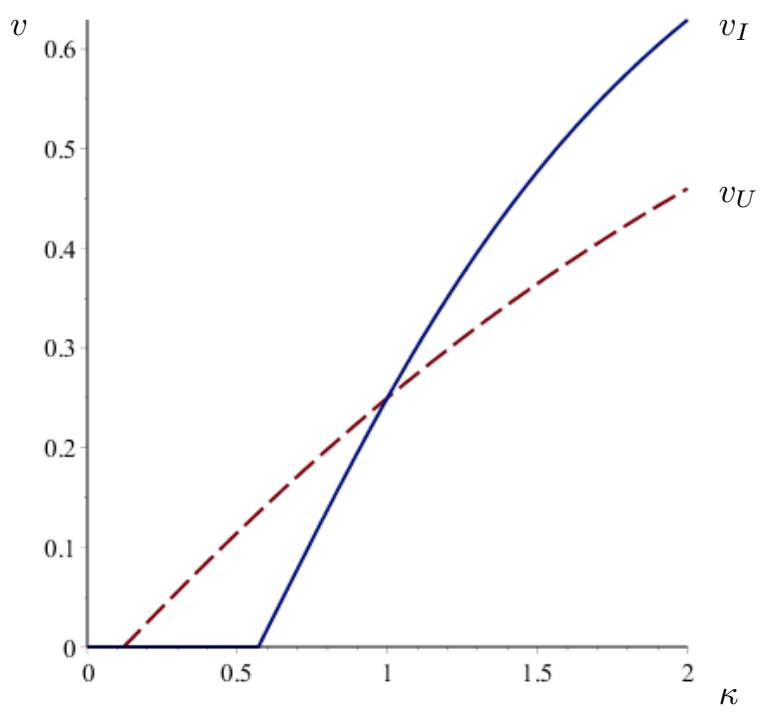

Figure 2: Incentive rates with perfect and uninformative systems

Numerical example with $\alpha=0.5, E[\theta]=0.5, \operatorname{Var}(\theta)=3, k_{t}=1, k_{r}=\kappa \cdot k_{t}$ Solid (dashed) line represents the incentive rate with perfect (uninformative) system

Corollary 3 The optimal incentive rates $v_{I}$ and $v_{U}$ are strictly increasing in $\kappa$ and it holds that $v_{I} \gtreqless v_{U}$ if and only if $\kappa \gtreqless 1$.

As seen from Lemma 1, the optimal incentive rates are decreasing functions of $\alpha$ and $\rho_{i}$. Since the relative importance of real effects $(\alpha)$ is not affected by the information system in place, the difference in incentive rates can only be caused by the difference in the relative productivities as measured by $\rho_{i}$. While $\rho_{U}=t(E[\theta]) / r(E[\theta])$ is a function of the prior expectation about the productivity parameter, $\rho_{I}=\sqrt{E\left[t(\theta)^{2}\right] / E\left[r(\theta)^{2}\right]}$ also considers the variance in the productivities because the productivity parameter $\theta$ is unknown at the contracting stage. Moreover, since $E\left[i(\theta)^{2}\right]-i(E[\theta])^{2}=\operatorname{Var}(i(\theta))$ for $i \in\{r, t\}$, this variance increases both the principal's expected benefits and costs from providing the agent with information about $\theta$. In fact, using the definition of $\rho_{I}^{2}$ it is easy to see from the expected surplus expression in (16) that the principal benefits from $\operatorname{Var}(r(\theta))$ but suffers from $\operatorname{Var}(t(\theta))$ because the agency cost is decreasing in the former and increasing in the latter case. Intuitively, providing information about 
$\theta$ increases the agent's expected contribution to firm value in the former case and the expected agency cost in the latter case.

Evaluating the relative magnitude of the incentive rates requires a comparison of the expected productivities for both information regimes. Straightforward algebra shows that the incentive rate under perfect information is higher than the one under no information if:

$$
\frac{k_{r} \sqrt{\operatorname{Var}(\theta)}}{1+k_{r} E[\theta]}>\frac{k_{t} \sqrt{\operatorname{Var}(\theta)}}{1+k_{t} E[\theta]} .
$$

Put differently, if the relative dispersion of the value creation productivity is larger than the relative dispersion of the manipulation productivity, the principal benefits from providing the agent with a higher PPS because providing the agent with information

mitigates the threat of manipulation and reduces the agency cost. If the opposite holds, providing the agent with information exacerbates the manipulation threat and increases the agency cost. Therefore, the principal sets a higher incentive rate with an uninformative system if condition (17) is violated. It follows from the definition of $\kappa$ that $v_{I} \gtreqless v_{U}$ if and only if $\kappa \gtreqless 1$.

\subsubsection{Equilibrium}

As a final step we consider which of the two solutions to the principal's simultaneous information design and contracting problem is globally optimal. Let $v^{*}$ denote the equilibrium PPS.

Proposition 2 If $\alpha \geq 1$ the principal offers $v^{*}=v_{I}$ for any $\kappa \in[\underline{\kappa}, \bar{\kappa}]$ and implements a perfect information system. If $\alpha<1$ there exists a unique threshold value $\widehat{\kappa} \in(\underline{\kappa}, 1)$ such that:

(i) If $\kappa \in[\underline{\kappa}, \widehat{\kappa})$, the principal offers $v^{*}=v_{U}$ and implements an uninformative system.

(ii) If $\kappa \in[\widehat{\kappa}, \bar{\kappa}]$, the principal offers $v^{*}=v_{I}$ and implements a perfect information system. 
The range of productivity ratio $[\widehat{\kappa}, \bar{\kappa}]$ for which the principal implements a perfect information system is increasing in $\alpha$.

The result of Proposition 2 is graphically illustrated in Figure 3. A consistent choice of $v$ and $g(\cdot)$ requires that all constraints are satisfied. That is, an uninformative system can only be optimal if $v_{U}>\widehat{v}$ and a perfect information system can only be optimal if $v_{I} \leq \widehat{v}$. Since $\widehat{v}>v_{I}$ if $\kappa>1$ but $v_{I}>v_{U}$, it is easy to see that the principal always strictly prefers a perfect information system if $\kappa>1$. This solution is quite intuitive because, for mild manipulation threat $(\kappa>1)$, more precise information about $\theta$ increases the principal's expected benefit from allowing the agent to tailor his productive decision to the actual productivity without imposing too high of a cost from earnings management. In fact, it can be shown that in this parameter region the difference between $\widehat{v}$ and $v_{I}$ is strictly positive and increasing in $\kappa$. Since the distance between $\widehat{v}$ and $v_{I}$ scales the impact of the conditional variance on the principal's expected profit and thereby her expected benefits from providing the agent with more precise information, higher values of $\kappa$ make the provision of information more attractive to the principal.

Lower values of $\kappa$ have the opposite effect on the principal's expected profit. In particular, whenever $\kappa<1$, information about $\theta$ exacerbates the agent's manipulation tendency. Moreover, lower values of $\kappa$ reduce the expected benefit from allowing the agent to tailor his productive activity. However, since the total surplus is strictly convex in $E[\theta \mid \sigma]$ if $\kappa=1$ and $\alpha<1$, the principal still prefers to implement a perfect information system provided that $1>\kappa>\widehat{\kappa}$ and $\alpha<1$. If $\kappa<\widehat{\kappa}$ the principal strictly prefers to implement an uninformative system because the benefit derived from tailoring the agent's production to the true productivity is outweighed by the increasing manipulation cost. In this case, it holds that $\widehat{v}<v_{U}$ so that providing information to the agent reduces the principal's expected profit. Accordingly, the principal does best by implementing an uninformative system.

Note that the second solution can never be optimal if the agent's earnings management activities have a significantly negative impact on firm value $(\alpha \geq 1)$. Then, the 


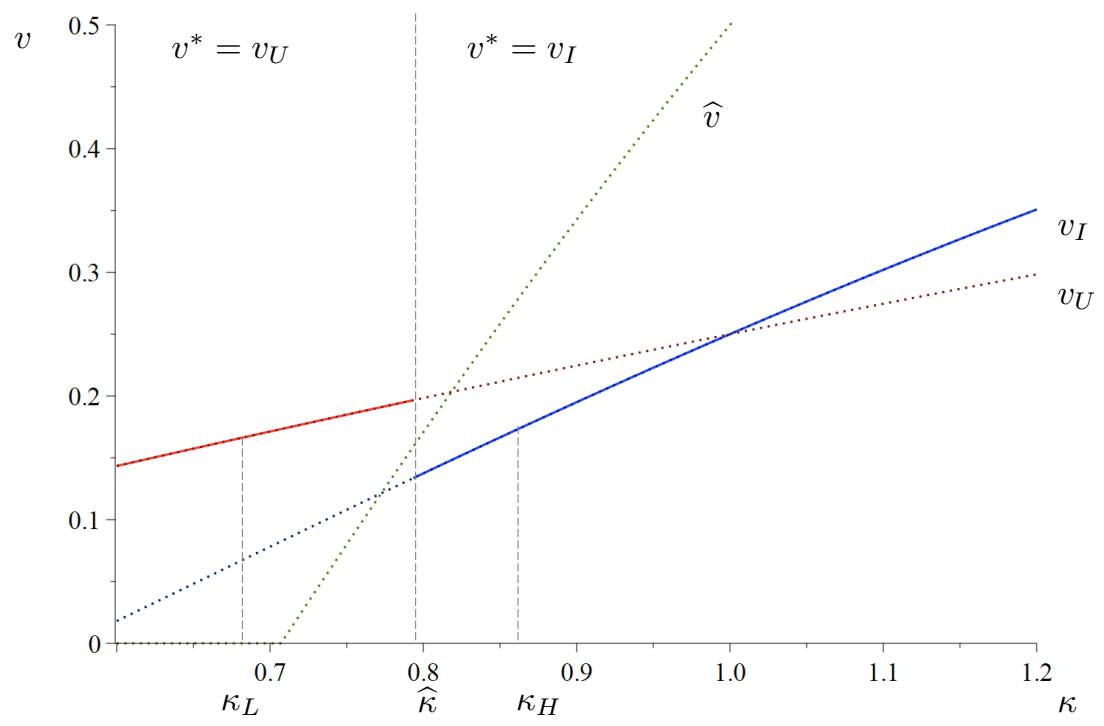

Figure 3: Optimal incentive rates

Numerical example with $\alpha=0.5, E[\theta]=0.5, \operatorname{Var}(\theta)=3, k_{t}=1, k_{r}=\kappa \cdot k_{t}$ Solid line represents the equilibrium incentive rate. Here, $\widehat{\kappa}=0.79$.

principal always provides the agent with perfect information about $\theta$ despite the fact that the agent's earnings management have severe financial consequences. The reason is that, for $\alpha \geq 1$, the principal offers $v>0$ only if $\kappa>1$ because, otherwise, a positive incentive rate would destroy more value than it creates. However, if $\kappa>1$, it holds that $\widehat{v}>v_{I}>v_{U}$ so that the total surplus is always strictly convex in $E[\theta \mid \sigma]$.

Corollary 4 The optimal pay-performance sensitivity $v^{*}$ is increasing in $\kappa$ for any $\kappa<\widehat{\kappa}$ and $\kappa>\widehat{\kappa}$ but $\lim _{\kappa \rightarrow \widehat{\kappa}^{-}} v^{*}>\lim _{\kappa \rightarrow \widehat{\kappa}^{+}} v^{*}$. Furthermore, there exist $\kappa_{L} \in[0, \widehat{\kappa})$ and $\kappa_{H} \in(\widehat{\kappa}, 1)$ such that:

(i) For any $\kappa<\kappa_{L}$ the principal implements an uninformative system and offers a PPS that is strictly lower than the PPS she offers when $\kappa>\kappa_{H}$ and she implements a perfect information system.

(ii) For any $\kappa \in\left(\kappa_{L}, \widehat{\kappa}\right]$ the principal implements an uninformative system and offers 
a PPS that is strictly higher than the PPS she offers when $\kappa \in\left(\widehat{\kappa}, \kappa_{H}\right)$ and she implements a perfect information system.

When $\kappa=0$ the principal prefers to implement a system that provides no information and offers a PPS of $v_{U}$. This is because if $\kappa=0$ then $k_{r}=0$, i.e., information does not help with value creation but only exacerbates the earnings manipulation. As $\kappa$ increases, the manipulation threat becomes less severe. As shown in Figure 3, while the principal still prefers to provide no information, the conditionally optimal incentive rate $v_{U}$ increases because $k_{r}$ is now strictly positive and higher PPS will encourage value creation.

At some critical value $\widehat{\kappa}$ the principal finds it optimal to switch to a perfect information system and offer the conditionally optimal pay-performance sensitivity $v_{I}$. Because $\widehat{\kappa}$ is strictly lower than 1 (the value at which the two conditionally optimal incentive rates intersect), it holds that $v_{U}<v_{I}$ at $\widehat{\kappa}$ so that $v^{*}$ drops from a level of $v_{U}$ to a level of $v_{I}$. Hence, there is always a set $\kappa \in\left(\widehat{\kappa}, \kappa_{H}\right)$ of firms that optimally combine a perfect information system with a low PPS and a second set of firms $\kappa \in\left(\kappa_{L}, \widehat{\kappa}\right]$ that optimally combine an uninformative system with a higher PPS. Notably, even though the performance measure of firms in $\kappa \in\left(\kappa_{L}, \widehat{\kappa}\right]$ is more prone to gaming than for those in $\kappa \in\left(\widehat{\kappa}, \kappa_{H}\right)$, the former group of firms optimally provides higher-powered incentives than the latter. In summary, our results imply that: (i) information design and financial incentives may be substitutes in solving the firm's incentive problem and (ii) the combined optimal use of information and incentives in solving the underlying agency problem can surprisingly render it optimal to provide the agent with higher PPS if the performance measure is more prone to gaming. This offers a qualification of the predictions made by prior literature studying optimal incentives for a given information environment (e.g., Beyer, Guttman and Marinovic 2014). Our results suggest that future empirical studies could include proxies for information environment and manipulation threat. 


\section{Model variations}

\subsection{The role of internal controls}

The analysis of section 3 focuses on the interplay between information design and monetary compensation in balancing the agent's incentives to create firm value and to manage earnings. Frequently, firms also use their internal control system to increase the reliability of their financial reporting. To allow for this possibility we assume that a given level of internal control effort $e$ raises the agent's marginal cost of earnings management by the amount $c e$. Establishing an internal control system comes at a cost $Q(e)$ which is quadratic with $Q(0)=0$ and $Q^{\prime \prime}(e)=n$. To avoid trivial solutions where the internal control system completely discourages the agent's earnings management activities, we assume that $n$ is sufficiently large. ${ }^{25}$

To analyze how the internal control system affects the solution of the agency problem, we consider two different scenarios. First, we study the case where the principal commits to the level of internal control along with her choice of the information system and the PPS of the agent's contract at date 2. Second, we also study the case where the principal sets the control level after observing the signal about the unknown parameter. If the principal decides on the control level before observing the signal, she must fix $e$ based on her prior expectations about the marginal productivity in managing the firm's earnings. In contrast, if the principal sets $e$ after observing $\sigma$, she can tailor the internal control system to the updated information about the marginal productivity.

Proposition 3 If the principal commits to a control system at date 2 , she chooses a control level of $\bar{e}=\alpha \cdot E[t(\theta)] / n$ which is independent of the information system and incentive rate choices at date 2.

If the level of internal control is chosen early, it is independent of the other decisions taken at date 2, i.e., information system and incentive rate. Accordingly, the principal adopts the same policy as defined in Proposition 3. The reason for this result is twofold.

\footnotetext{
${ }^{25}$ The minimal $n$ that supports a positive manipulation in equilibrium is provided in the proof of Lemma 3.
} 
First, the principal commits to the control level before the information system generates a signal and so the control level only enters the total surplus as a linear term. Hence, the curvature of the total surplus is independent of the control level so that the condition for the optimal choice of the information system in Proposition 1 remains the same. Second, the choice of $e$ is independent of $v$ because the agent corrects his manipulation by the control level $e$. In fact, in equilibrium, the agent chooses manipulation level of $a_{t}^{o}(v, e, \sigma)=v \cdot E[t(\theta) \mid \sigma] / c-e$, which is additively separable in $e$ and $v$. This structure implies that, in equilibrium, the manipulation cost $C\left(a_{t}^{o}(v, e, \sigma, g(\cdot))+e\right)=C\left(a_{t}^{o}(v, \sigma)\right)$ is independent of the optimal control level and identical to the manipulation cost in the baseline model. The principal benefits from the internal control system because the agent reduces the level of earnings management. In equilibrium, this reduction equals $\bar{e}$ and the principal increases her expected profit by the amount $\alpha E[t(\theta)] \bar{e}-Q(\bar{e})=$ $\frac{\alpha^{2}}{2 n}\left(1+k_{t} E[\theta]\right)^{2}$.

Now consider a scenario in which the principal chooses the internal control level at date 3 , after $\sigma$ is realized. Then, the level of internal control is no longer independent of her other choices.

Lemma 2 If the principal chooses the internal control system at date 3, she chooses a level of $e^{o}=(\alpha+v) \cdot E[t(\theta) \mid \sigma] / n$ which is strictly higher than the socially optimal level $e^{*}=\alpha \cdot E[t(\theta) \mid \sigma] / n$.

If the principal delays the choice of internal control level until the signal about $\theta$ is observed, she can use the information to tailor the control level to the actual manipulation productivity. Specifically, the principal's choice then is given by $e^{o}=$ $(\alpha+v) \cdot t(\theta) / n$. However, this level is strictly higher than the level of control that maximizes the total surplus of the agency for a perfect information system at date 3, $e^{*}=\alpha \cdot t(\theta) / n$. To understand why the principal's control level exceeds the social optimum, recall that the agent's contract is set at date 2. At the contracting stage, the principal anticipates that the agent's equilibrium manipulation level is decreasing in the level of control and increases the fixed component of pay accordingly. ${ }^{26}$ However, when

\footnotetext{
${ }^{26}$ In fact, it is easy to see that $w=E_{\sigma}\left[C\left(a_{r}^{o}(v, \sigma)\right)+C\left(a_{t}^{o}(v, e, \sigma)+e\right)-v a_{t}^{o}(v, e, \sigma)\right]$ is increasing in
} 
deciding on the level of control at date 3, the principal ignores the positive effect of the expected control level on the fixed component of the agent's pay because $w$ is sunk cost from the principal's perspective at this point. Therefore, the principal chooses a level of late control that exceeds the social optimum. Interestingly, this result holds not only under perfect information system as described above, but also under uninformative one. Then, the optimal late control level is $e^{o}=(\alpha+v) \cdot E[t(\theta)] / n$ which is higher than the socially optimal control level $e^{*}=\alpha \cdot E[t(\theta)] / n=\bar{e}$. Put differently, for a given information system in place, the amount by which late control level exceeds the social optimum is $e^{o}-e^{*}=v \cdot E[t(\theta) \mid \sigma] / n$.

Lemma 3 Suppose the principal chooses the internal control system at date 3.

(i) For a given incentive rate $v$, the critical rate below which the principal prefers a perfect information system is given by $\widehat{v}^{e}$, where it holds that $\widehat{v}^{e} \leq \widehat{v}$ if $\alpha \geq \frac{2 \kappa^{2}}{\kappa^{2}+3}$ and $\widehat{v}^{e}>\widehat{v}$ if $\alpha<\frac{2 \kappa^{2}}{\kappa^{2}+3}$.

(ii) For a given information system, the principal offers the conditionally optimal incentive rate

$$
v_{j}^{e}=\frac{1-\alpha \rho_{j}^{2}}{1+\left(1+\frac{c}{n}\right) \rho_{j}^{2}}, \quad j \in\{U, I\}
$$

where it holds that $v_{j}^{e}<v_{j}$.

Anticipating the optimal level of control implemented at date 3, the principal's date 2 profit becomes

$$
\Gamma(v)+\frac{\alpha^{2}-v^{2}}{2 n} \cdot\left[\left(1+k_{t} E[\theta]\right)^{2}+k_{t}^{2} \cdot \operatorname{Var}(E[\theta \mid \sigma])\right]
$$

where $\Gamma(v)$ is the expected surplus in the baseline model without internal control and the second term is the net effect of control on the firm's date 2 profit. Since the additional profit component is a function of $v$ and $\operatorname{Var}(E[\theta \mid \sigma])$, the choices of the optimal PPS and the information system are generally affected by the expected consequences of the $e$ because $a_{t}^{o}(v, e, \sigma)=v E[t(\theta) \mid \sigma] / c-e$. 
control activity undertaken at date 3 unless it happens to be that $\alpha=v$ so that the second term in (18) vanishes.

Consider now the optimal PPS for a given information system in Lemma 3. Since the additional profit component is monotonically decreasing in $v$, it must be that the optimal PPS for a given information system is decreasing with late control. This is because the principal controls too much ex post and the only way to balance this effect is to set a PPS lower than the rate that maximizes $\Gamma(v)$.

The effect of control on the critical value $\widehat{v}^{e}$ below which the principal prefers a perfect information system is more subtle because the additional profit is increasing in $\operatorname{Var}(E[\theta \mid \sigma])$ if $\alpha>v$ but decreasing if $\alpha<v$. Accordingly, the presence of late internal control makes a higher posterior variance relatively more desirable if the cost of real earnings management is more important for the firm's profit than the marginal cost of rewarding the agent for his manipulation activities and vice versa. As a consequence, the firm is less willing to provide the agent with access to perfect information if $\alpha<\widehat{v}$ (which is equivalent to $\alpha>\frac{2 \kappa^{2}}{\kappa^{2}+3}$ ) and more willing to inform the agent if $\alpha>\widehat{v}$ (which is equivalent to $\left.\alpha<\frac{2 \kappa^{2}}{\kappa^{2}+3}\right)$. Therefore it holds that $\widehat{v}^{e}<\widehat{v}$ in the former case and $\widehat{v}^{e}>\widehat{v}$ in the latter case.

Comparing the expected surplus of the principal under early control with that under late control immediately, for given information system, yields the following result:

\section{Proposition 4}

(i) With an uninformative system in place, the principal strictly prefers early over late control.

(ii) With a perfect information system in place, the principal prefers late control if the following condition holds

$$
\left(\frac{\alpha}{v_{I}^{e}}\right)^{2}>1+\frac{E[\theta]^{2}}{k_{t}^{2} \operatorname{Var}(\theta)},
$$

where $v_{I}^{e}$ depends on exogenous parameters and is defined in Lemma 3. 
While, by revealed preference, internal control benefits the principal, she may prefer to commit to the control level early, before observing the signal about $\theta$. This is because with late control the principal controls too much at date 3 . Under a perfect information system, whenever the real effects of earnings management are sufficiently high, the additional profit derived from internal control is determined by the cost of excessive control and the informational benefits of tailoring the control level to the true productivity. This trade-off is reflected in condition (19). The higher $\alpha$ relative to the optimal incentive rate $v_{I}^{e}$, the higher the benefits of avoiding the real effects of earning management relative to the additional compensation cost caused by the manipulation threat. On the other hand, a higher variance of $\theta$ increases the expected benefit of tailoring the optimal control level to the true productivity and makes late control more beneficial for the principal.

Proposition 5 There exists $\bar{\alpha} \leq 1$ such that, in equilibrium, the principal chooses a perfect information system if $\alpha>\bar{\alpha}$ for any $\kappa$. For $\alpha<\bar{\alpha}$ there exists a critical value of $\widehat{\kappa}^{e} \in[\underline{\kappa}, 1)$ so that principal chooses a perfect information system and the incentive rate $v_{I}^{e}$ if $\kappa \geq \widehat{\kappa}^{e}$ and an uninformative system with the incentive rate $v_{U}^{e}$ if $\kappa<\widehat{\kappa}^{e}$.

Combining the information design and incentive rate choices yields the same equilibrium structure as in Proposition 2. However, unlike the baseline model without internal control, the principal now finds it optimal to choose a perfect information system regardless of the agent's relative productivity level $\kappa \geq \underline{\kappa}$ whenever $\alpha$ is sufficiently high (above some $\bar{\alpha} \leq 1$ ). The reason is that the real effects of earnings management not only determine the magnitude of the PPS of the agent's contract but also the principal's benefit from an informed internal control. Since severe real effects reduce the optimal PPS but increase the marginal benefit of informed control, the principal finds it optimal to choose a perfect information system regardless of the relative marginal productivity if $\alpha$ is sufficiently high. 


\subsection{The role of authority over information design}

So far, we assumed that the principal is in charge of designing both the firm's information system and the agent's compensation contract. In this section we briefly study how the results of the previous sections change if the agent is in charge of the information design decision at date 2. Specifically, we now solve for a Nash-equilibrium at date 2 in which the agent chooses $g(\cdot)$ and the principal chooses $v$. In a richer model structure where a board of directors or a specialized compensation committee takes the role of the principal and the agent is the CEO, this setting represents a scenario where the board retains the right to decide on the CEO's compensation contract but the decision on the design of the firm's information system is in the hands of the CEO.

Proposition 6 Consider the baseline model without internal control. If the agent is in charge of the information design, he always implements a perfect information system and the principal offers incentive rate $v_{I}$ as defined in Lemma 1.

Different from our result in Proposition 1, the agent always finds it optimal to implement a perfect information system at date 2. This result is independent of the magnitude of the PPS set by the principal at date 2. Intuitively this result stems from the fact that the agent not only benefits from tailoring his value creating activity but also his manipulation to the true productivity because both activities increase the agent's expected compensation. As a consequence, the agent's date 3 utility is an increasing and convex function of the conditional expectation $E[\theta \mid \sigma]$ which implies, by Jensen's inequality, that the agent strictly benefits from an informed choice of both activities. Thus, in contrast to the principal, the agent always strictly benefits from a perfect information system regardless of the relative productivity in performing the two activities.

Proposition 7 Consider the internal control model in section 4.1.

(1) With early control at date 2, the agent always implements a perfect information system and the principal sets a control level $\bar{e}$ as defined in Proposition 3 and an incentive rate $v_{I}$ as defined in Lemma 1. 
(2) With late control at date 3, there exists a critical value

$$
\widehat{v}_{D}=\frac{2 \alpha}{\frac{n}{c}\left(1+\kappa^{2}\right)-1}
$$

such that, for $v_{j}^{e}, j=I, U$ as defined based on exogenous parameters in Lemma 3:

(i) The agent implements a perfect information system and the principal sets the optimal incentive rate $v_{I}^{e}$ if $v_{I}^{e} \geq \widehat{v}_{D}$.

(ii) The agent implements an uninformative system and the principal sets the optimal incentive rate $v_{U}^{e}$ if $\widehat{v}_{D}>v_{U}^{e}$.

(iii) If $v_{U}^{e} \geq \widehat{v}_{D}>v_{I}^{e}$, the agent implements a perfect information system with probability $\lambda \in(0,1)$ and an uninformative system with probability $1-\lambda$. The principal offers $v=\widehat{v}_{D}$.

(3) The optimal incentive rate in the mixed-strategy region is monotonically decreasing in $\kappa$.

If the principal commits to an internal control system at date 2 , this choice has no consequences for the agent's information design problem. Because the principal cannot condition her optimal control level on the true productivity in manipulating the firm's earnings, the agent unambiguously benefits from tailoring both activities to the true productivity and strictly prefers a perfect information system.

However, if the principal chooses the level of control at date 3 , the agent implements a perfect information system only if his PPS is sufficiently high. Otherwise, he either strictly prefers an uninformative system or chooses an uninformative system with positive probability. This result stands in sharp contrast to the principal's optimal policy who, ceteris paribus, prefers a perfect information system for low incentive rates. This difference can best be understood by considering the dual role of information in the late control scenario. On the one hand, the agent can increase his expected utility by adjusting his decisions to the economic environment. On the other hand, precise information allows the principal to implement a more effective control strategy, which 


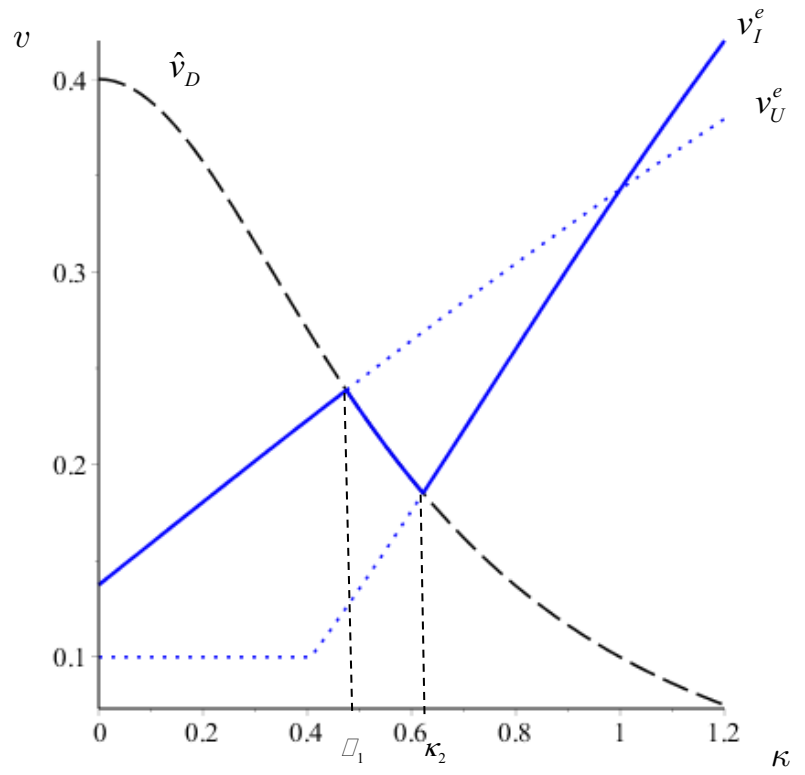

Figure 4: Equilibrium with late control and agent's authority

Numerical example with $\alpha=0.2, E[\theta]=0.5, \operatorname{Var}(\theta)=3, k_{t}=1, k_{r}=\kappa \cdot k_{t}, c=1, n=3$.

Solid line represents the equilibrium incentive rate

in turn reduces the agent's expected utility. In fact, since an effective control strategy reduces the agent's expected compensation and the reduction caused by internal control is a convex function of the conditional expectation, the agent prefers less often a perfect information system than in the absence of control.

Figure 4 illustrates, with a numerical example, the equilibrium in the late control case. The piecewise solid line represents the optimal incentive rates along the equilibrium path. If the manipulation threat is relatively severe $\left(\kappa<\kappa_{1}\right)$, the condition in Proposition $7(2)(\mathrm{ii}), \widehat{v}_{D}>v_{U}^{e}$, is satisfied, and so the agent implements an uninformative system and the principal sets an incentive rate of $v_{U}^{e}$. Intuitively, the agent prefers not to gather information about the productivity if he is relatively more productive in earnings manipulation than in value creation because in this case, the agent benefits less from tailoring his production action to the true productivity $r(\theta)$ and suffers more from allowing the principal to tailor his control level to the true manipulation productivity $t(\theta)$. If the manipulation threat becomes less pronounced $\left(\kappa_{1} \leq \kappa<\kappa_{2}\right)$, corresponding 
to the condition in Proposition 7(2)(iii), $v_{U}^{e} \geq \widehat{v}_{D}>v_{I}^{e}$, the agent adopts a mixed strategy and implements a perfect information system with probability $\lambda$ and the principal offers $\widehat{v}_{D}$. Finally, if the manipulation threat is very mild $\left(\kappa \geq \kappa_{2}\right)$, corresponding to the condition in Proposition 7(2)(i), $v_{I}^{e} \geq \widehat{v}_{D}$, the agent implements a perfect information system and the principal sets the incentive rate $v_{I}^{e}$.

As in the baseline model, the optimal PPS is a non-monotonic function of the agent's optimal information design choice. Particularly, in the region of the mixed strategy equilibrium the optimal PPS is monotonically decreasing in $\kappa$ because a less severe manipulation threat lowers $\widehat{v}_{D}$ keeping the agent indifferent between choosing a perfect and an uninformative system. Thus, if the agent is in charge of the information design, there is always a non-empty set of agencies (in the numerical example represented by $\left.\kappa \in\left[\kappa_{1}, \kappa_{2}\right]\right)$ for which the PPS of the optimal contract is increasing as the performance measure becomes more prone to manipulation.

\section{Conclusion}

We consider a model where a firm manager is compensated on the basis of a performance measure that is affected by his productive and unproductive "window dressing" activities. The firm has authority over the implementation of an information system that will generate decision-relevant information. Because of the multi-task problem, the firm faces a trade-off between (i) the benefits from allowing the manager to learn information about the productivity in creating value, and (ii) the cost of allowing him to learn about the productivity in manipulating earnings.

We identify conditions under which the implementation of a system that provides perfect information is no longer optimal for the firm and it prefers not to implement an information system. Notably, we identify conditions under which the information system and the pay-performance-sensitivity of the optimal compensation contract are substitutes in solving the firm's incentive problem in the sense that the firm optimally combines a perfect information system with a low PPS or an uninformative system with a high PPS. We show that the optimal combination of information design and financial 
incentives may render it optimal to provide agents with high-powered incentives as the performance measure of the compensation contract becomes more prone to gaming. We also study the interplay between the firm's information and internal control systems when the choice of the optimal control level can be made before or after the manager observes any information. While internal control is always beneficial, the firm may prefer to commit early to the control level in order to avoid overinvesting in control ex post. Finally, we find that if the manager decides on the information design, he prefers perfect information unless the firm uses the information for internal control.

Our results shed a new light on the mixed evidence about the empirical association between financial incentives and measures of earnings management. The model suggests that the information environment in which firms operate as well as how prone are their performance measures to manipulation could explain the cross-sectional variation among firms. Future empirical studies analyzing this relationship could exploit our predictions by including proxies for the information environment and the manipulation threat of different firms and industries into their empirical research design. 


\section{Appendix A: Notations and proofs}

\begin{tabular}{ll}
\hline Variable & Definition \\
\hline$x$ & firm's terminal value \\
$y$ & firm's earnings \\
$s(y)=w+v \cdot y$ & incentive contract \\
$v$ & incentive rate or pay-performance sensitivity (PPS) \\
$\alpha$ & severity of the real effects of earnings manipulation \\
$a_{r}$ & productive effort/activity (value creation) \\
$a_{t}$ & unproductive effort/activity (earnings manipulation) \\
$C\left(a_{i}\right)$ & cost of activity $i=r, t$ \\
$i\left(\phi_{i}\right)=k_{0}+k_{i} \phi_{i}$ & marginal productivity of activity $i=r, t$ \\
$\kappa=k_{r} / k_{t}$ & level of manipulation threat (low $\kappa$ indicates severe threat) \\
$\phi_{i}=\theta+\xi_{i}$ & parameter determining marginal productivity of $a_{i}, i=r, t$ \\
$\xi_{i}$ & random shock affecting activity $i=r, t$ \\
$\theta$ & common parameter affecting both activities \\
$\sigma$ & signal about $\theta$ generated by the information system \\
$F(\theta)$ & prior cumulative distribution function $(\mathrm{CDF})$ of $\theta$ \\
$f(\theta)$ & prior probability density function (PDF) of $\theta$ \\
$G(\theta \mid \sigma)$ & cumulative distribution function $(\mathrm{CDF})$ of posterior $\theta \mid \sigma$ \\
\hline \hline &
\end{tabular}

Table A.1: Notations for the main model 
Proof of Proposition 1: Using (10) and (11), the principal maximizes

$$
\begin{aligned}
\Gamma(v) & =E\left[x^{o}(v, \sigma)-\alpha\left(y^{o}(v, \sigma)-x^{o}(v, \sigma)\right)-\sum_{i=r, t} C\left(a_{i}^{o}(v, \sigma)\right)\right] \\
& =E\left[\frac{v}{c}\left(\left(1-\frac{v}{2}\right)\left(1+k_{r} E[\theta \mid \sigma]\right)^{2}-\left(\alpha+\frac{v}{2}\right)\left(1+k_{t} E[\theta \mid \sigma]\right)^{2}\right)\right]
\end{aligned}
$$

Now note that $E\left[\left(1+k_{i} E[\theta \mid \sigma]\right)^{2}\right]=\left(1+k_{i} E[\theta]\right)^{2}+k_{i}^{2} \operatorname{Var}(E[\theta \mid \sigma])$ for $i \in\{r, t\}$ which allows us to restate the problem as of maximizing

$$
\begin{aligned}
\Gamma(v)= & \underbrace{\frac{v}{c}\left(1-\frac{v}{2}\right)\left(1+k_{r} E[\theta]\right)^{2}-\frac{v}{c}\left(\alpha+\frac{v}{2}\right)\left(1+k_{t} E[\theta]\right)^{2}}_{=\text {const }} \\
& +\frac{v}{c}\left(\left(1-\frac{v}{2}\right) \cdot k_{r}^{2}-\left(\alpha+\frac{v}{2}\right) \cdot k_{t}^{2}\right) \operatorname{Var}(E[\theta \mid \sigma]) \\
= & \text { const }+\frac{v}{c} \cdot \frac{\left(k_{r}^{2}+k_{t}^{2}\right)}{2}(\widehat{v}-v) \cdot \operatorname{Var}(E[\theta \mid \sigma]) .
\end{aligned}
$$

Hence, if $v \leq \widehat{v}$, the principal's expected payoff is increasing in the variance of posterior expectations and the principal will choose a fully-informative system with $\sigma=\theta$. Otherwise, the principal will choose uninformative system.

Proof of Corollary 3: From Lemma 1, the optimal incentive rate with perfect information is $v_{I}=\frac{1-\alpha \rho_{I}^{2}}{1+\rho_{I}^{2}}$ and with uninformative system is $v_{U}=\frac{1-\alpha \rho_{U}^{2}}{1+\rho_{U}^{2}}$. Comparing these expression, we can see that $v_{I} \gtreqless v_{U}$ if $\rho_{U}^{2} \gtreqless \rho_{I}^{2}$, where $\rho_{i}^{2}, i \in\{U, I\}$ measures the relative magnitude of the agency cost in the two information scenarios. Evaluating the relevant expressions yields

$$
\rho_{U}^{2}=\frac{t(E[\theta])^{2}}{r(E[\theta])^{2}}, \quad \rho_{I}^{2}=\frac{E\left[t(\theta)^{2}\right]}{E\left[r(\theta)^{2}\right]}=\frac{t(E[\theta])^{2}+\operatorname{Var}(t(\theta))}{r(E[\theta])^{2}+\operatorname{Var}(r(\theta))} .
$$

Using the facts that $\operatorname{Var}(i(\theta))=k_{i}^{2} \operatorname{Var}(\theta)$ for $i \in\{r, t\}$ and $\kappa=k_{r} / k_{t}$, it follows that both incentive rates are increasing in $\kappa$ and that $v_{I} \gtreqless v_{U}$ if and only if $\kappa \gtreqless 1$.

Proof of Proposition 2: Given that, by Corollary 3, $v_{I} \gtreqless v_{U}$ iff $\kappa \gtreqless 1$ and $\widehat{v} \in[0,1]$ for $\kappa \in[\underline{\kappa}, \bar{\kappa}]$ we conclude that at least one of the two conditions $v_{I} \leq \widehat{v}$ or $v_{U}>\widehat{v}$ is always met.

We can distinguish three different cases. First, if $\kappa \geq 1$ it easy to verify that $\widehat{v}>v_{I}$ and since $v_{I}>v_{U}$ in this case, the optimal solution is to provide perfect information and choose an incentive rate of $v_{I}$ if $\kappa>1$. This solution is also optimal if $\kappa<1$ as long as $\widehat{v}>v_{U}>v_{I}$. However, there are two more cases to consider. If $v_{U}>v_{I}>\widehat{v}$, the optimal solution is $v_{U}$ and the principal implements an uninformative system. However, if $v_{U}>\widehat{v}>v_{I}$ both conditions are met. In this case, the principal chooses the incentive 
rate that yields the largest expected profit. Let $\Gamma(v)$ denote the principal's objective evaluated at an incentive rate $v$. If $v_{U}>\widehat{v}>v_{I}$ and $\Gamma\left(v_{U}\right)>\Gamma\left(v_{I}\right)$, the firm chooses $v_{U}$ and leaves the agent uninformed, whereas if $v_{U}>\widehat{v}>v_{I}$ and $\Gamma\left(v_{U}\right)<\Gamma\left(v_{I}\right)$, the firm chooses a perfect information system and sets the incentive rate $v_{I}$.

The relevant solution depends on the values of $\kappa \in(\underline{\kappa}, \bar{\kappa})$ and $\alpha \geq 0$. Consider now the case where $\alpha \geq 1$. Since $\underline{\kappa}=\sqrt{\alpha} \geq 1$ for this case, it must be that $\widehat{v}>v_{I}>$ $v_{U}$. Thus, if earnings management has sufficiently high real effects, the firm optimally chooses a perfect information system and sets incentives $v_{I}$.

Consider next the case, where $\alpha<1$. If $\kappa \geq 1$ the solution is the same as for $\alpha=1$ but if $\kappa<1$, there is always a set of parameters for which the principal finds it optimal to choose the incentive rate $v_{U}$ and to leave the agent uninformed. To show existence, note that $\Gamma\left(v_{U}\right)=\Gamma\left(v_{I}\right)=0$ if $\alpha=0$ and $\kappa=\underline{\kappa}(0)=0$ because in this case it holds that $v_{U}=v_{I}=0$. However, if we evaluate profits and incentive rates for $\alpha \in(0,1)$ and $\kappa=\underline{\kappa}=\sqrt{\alpha}>0$. We find that

$$
\frac{v_{U}(\underline{\kappa})}{v_{I}(\underline{\kappa})}=\frac{\Gamma\left(v_{U}(\underline{\kappa})\right)}{\Gamma\left(v_{I}(\underline{\kappa})\right)}=1+\frac{\operatorname{Var}(\theta)}{k_{r}^{2} E[\theta]^{2}+\frac{2}{1+\alpha}\left(\underline{\kappa}(1+\underline{\kappa}) k_{r} E[\theta]+\alpha\right)}>1
$$

which allows us to conclude that $v_{U}(\underline{\kappa})>v_{I}(\underline{\kappa})$ and $\Gamma\left(v_{U}(\underline{\kappa})\right)>\Gamma\left(v_{I}(\underline{\kappa})\right)$ if $\alpha \in(0,1)$. Moreover, since $\widehat{v}(\underline{\kappa})=0$ for any $\alpha$, it holds that $v_{U}(\underline{\kappa})>v_{I}(\underline{\kappa})>\widehat{v}$. It follows that the firm optimally sets an incentive rate of $v_{U}$ and implements an uninformative system if $\alpha \in(0,1)$ and $\kappa=\underline{\kappa}$.

To determine $\widehat{\kappa}$ we first note that the incentive rates and equilibrium profits are monotonically increasing in $\kappa$ for $\kappa \in(\underline{\kappa}, \bar{\kappa})$. Moreover, since $v_{I}>v_{U}$ if $\kappa=1$, we know that for $\kappa=1$ it holds that

$$
\frac{\Gamma\left(v_{I}\right)}{\Gamma\left(v_{U}\right)}=\frac{v_{I}}{v_{U}} \cdot\left(1+\frac{\left(k_{r}^{2}+k_{t}^{2}\right) \operatorname{Var}(\theta)}{\left(1+k_{r} E[\theta]\right)^{2}+\left(1+k_{t} E[\theta]\right)^{2}}\right)>1 .
$$

Together with monotonicity, conditions (20) and (21) imply that there exists a unique intersection point $\kappa=\widehat{\kappa}<1$ so that the firm strictly prefers to leave the agent uninformed and sets $v=v_{U}$ if $\kappa<\widehat{\kappa}$ and to inform the agent perfectly and set $v=v_{I}$ if $\kappa \geq \widehat{\kappa}$.

Proof of Corollary 4: Follows immediately by Corollary 3 (comparative statics of $v_{j}$, $j=I, U)$ and Proposition $2(\widehat{\kappa}<1)$. The cutoffs $\kappa_{L}$ and $\kappa_{H}$ satisfy the equality

$$
\lim _{\kappa \rightarrow \kappa_{L}} v_{U}=\lim _{\kappa \rightarrow \kappa_{H}} v_{I}
$$

subject to $\kappa_{L} \in[0, \widehat{\kappa}]$ and $\kappa_{H} \in(\widehat{\kappa}, 1)$.

Proof of Proposition 3: With internal control, the agent's manipulation cost is 
$C\left(a_{t}+e\right)$ and the second incentive constraint in (4) becomes

$$
a_{t}^{o}(v, e, \sigma)=\frac{v}{c} \cdot E[t(\theta) \mid \sigma]-e
$$

Considering the modified incentive constraint and the principal's cost of control, the total surplus at date 3 can be written as

$$
\gamma(v, \sigma)+\alpha E[t(\theta) \mid \sigma] \cdot e-Q(e) .
$$

Since the second term in (24) is linear in $E[\theta \mid \sigma]$, the conditions for the firm's information choice in Proposition 1 are not affected by the choice of $e$. At date 2, the firm maximizes

$$
\Gamma(v)+\alpha\left(1+k_{t} E[\theta]\right) \cdot e-Q(e)
$$

with respect to $v, g(\cdot)$ and $e$. Since the objective function is additively separable, the optimal choices of solution of $v$, and $g(\cdot)$ are the same as in Proposition 2 and the firm implements an optimal control level so that $\alpha \cdot\left(1+k_{t} E[\theta]\right)=Q^{\prime}(e)$. With $Q(e)=n e^{2} / 2$, this condition yields the optimal control level $\bar{e}=\alpha \cdot\left(1+k_{t} E[\theta]\right) / n$.

Proof of Lemma 2: If the firm chooses $e$ after observing $\sigma$, the optimal control level maximizes the firm's profit at date 3 considering the agent's incentive constraints for productive activity in (4) and manipulation in (23). For a given signal $\sigma$ and given parameters of the compensation contract $s(y)$, this profit takes the form

$$
\Pi(\sigma)=(1-v) \cdot x^{o}(v, \sigma)-(\alpha+v) \cdot a_{t}^{o}(v, e, \sigma) \cdot E[t(\theta) \mid \sigma]-w-Q(e) .
$$

Maximizing $\Pi(\sigma)$ with respect to $e$ yields an optimal control level of $e^{o}=(\alpha+v)$. $E[t(\theta) \mid \sigma] / n$. In contrast, for a given information system, the socially optimal effort level maximizes the expression in (24) yielding the solution $e^{*}=\alpha \cdot E[t(\theta) \mid \sigma] / n$. Thus, whenever $v>0$, it holds that $e^{o}>e^{*}$.

Proof of Lemma 3: Anticipating the control level choice in Lemma 2, the principal's reduced form objective function at date 2 can be rewritten as

$$
\Gamma(v)+\frac{\alpha^{2}-v^{2}}{2 n} \cdot\left[\left(1+k_{t} E[\theta]\right)^{2}+k_{t}^{2} \cdot \operatorname{Var}(E[\theta \mid \sigma])\right] .
$$

Following the proof of Proposition 1, this expression can be rewritten as

$$
\propto \text { const }+\underbrace{\left(\frac{v\left(k_{r}^{2}+k_{t}^{2}\right)}{2 c}(\widehat{v}-v)+\frac{\alpha^{2}-v^{2}}{2 n}\right)}_{H(v, \alpha)} \cdot \operatorname{Var}(E[\theta \mid \sigma]) .
$$

To see how late control affects the optimal information system choice, recall that the sign 
of the expression in large brackets in $(26), H(v, \alpha)$, determines how the expected firm profit varies with the variance of the posterior expectation. Evaluating this expression at the critical incentive rate $v=\widehat{v}$ in the absence of control shows that the firm's expected profit at date 2 is increasing in $\operatorname{Var}(E[\theta \mid \sigma])$ at $v=\widehat{v}$ if $\alpha>\widehat{v}$ and decreasing in $\operatorname{Var}(E[\theta \mid \sigma])$ if $\alpha<\widehat{v}$. Let $\widehat{v}^{e}$ denote the cutoff-level for the firm's information system choice with late control, it follows that

$$
\widehat{v}^{e} \lesseqgtr \widehat{v} \quad \text { if } \quad \alpha \lesseqgtr \widehat{v}
$$

Substituting for $\widehat{v}$ yields the condition on exogenous parameters in the result. To derive a closed form expression for $\widehat{v}^{e}$ note that $H(v, \alpha)$ has one positive and one negative root. The relevant root is positive and takes the form

$$
\widehat{v}^{e}=\frac{1}{2(1+\tau)}\left(\widehat{v}+\sqrt{\widehat{v}^{2}+4 \tau(1+\tau) \cdot \alpha^{2}}\right), \quad \tau=\frac{c}{n k_{t}^{2}\left(1+\kappa^{2}\right)} .
$$

Maximizing $\Pi(\sigma)$ with respect to $v$ yields optimal incentive rates

$$
v_{j}^{e}=\frac{1-\alpha \rho_{j}^{2}}{1+\left(1+\frac{c}{n}\right) \rho_{j}^{2}}, \quad j \in\{U, I\}
$$

for a given information system $j \in\{U, I\}$. Since $n>c$, the incentive rates with late control are smaller than their counterparts without control in Proposition 2 but it still holds that $v_{I}^{e} \geq v_{U}^{e}$ if $\kappa \geq 1$ and $v_{I}^{e}<v_{U}^{e}$ if $\kappa<1$.

Proof of Proposition 4: The first part is straightforward. We know from Proposition 3 that $\bar{e}$ maximizes the expected date 2 surplus. On the other hand, Lemma 2 implies that $\bar{e}=e^{*}<e^{o}$, where $e^{o}=(\alpha+v) \cdot E[t(\theta)] / n$ and $e^{*}=\alpha \cdot t(E[\theta]) \mid / n$ for an uninformative system. Thus, since late control provides no informational benefits with an uninformative system, it strictly reduces the firm's profit because it induces an excessive level of control.

The second part is more subtle. With perfect information and late control, the firm chooses the control level $e^{o}=(\alpha+v) \cdot t(\theta) / n>\alpha \cdot t(\theta) / n=e^{*}$ that is perfectly adjusted to the state of nature. However, since the control level is set before the signal is observed, the control level with early control still equals $\bar{e}$. Evaluating the expected date 2 profits for both cases shows that late control is beneficial if

$$
\Gamma(v)+\frac{\alpha^{2}-v^{2}}{2 n} \cdot\left[\left(1+k_{t} E[\theta]\right)^{2}+k_{t}^{2} \cdot \operatorname{Var}(\theta)\right]>\Gamma(v)+\frac{\alpha^{2}}{2 n} \cdot\left(1+k_{t} E[\theta]\right)^{2} .
$$

Simplifying this expression considering that the optimal incentive rate with perfect information is $v_{I}^{e}$ yields the condition in (19). 
Proof of Proposition 5: The equilibrium has the same structure as in the baseline model but the role of $\alpha$ is different from the solution in Proposition 2. Since the proof of the equilibrium structure is similar to the proof of Proposition 2, we focus on the role of $\alpha$ in determining the equilibrium. As in the baseline model the firm strictly prefers a perfect information system if $\alpha=1$ because it holds that $\widehat{v}^{e}>\widehat{v}>v_{I}>v_{I}^{e}>v_{U}^{e}$ for this case. However, unlike the baseline model, the firm strictly prefers to install a perfect information system for all values $\kappa>\underline{\kappa}$ even if $\alpha<1$. For an uninformative system to be optimal it must be that $v_{U}^{e}>\widehat{v}^{e}$ for some $\kappa \in[\underline{\kappa}, 1)$ and $\alpha \in(0,1)$. Since the potential benefit of an uninformative system is the largest if $\kappa$ is low, it suffices to evaluate the term $H(v, \alpha)$ as defined in (26) at $v=v_{U}^{e}$ and $\underline{\kappa}=\sqrt{\alpha}$. Since $\widehat{v}=0$, and $k_{r}=\sqrt{\alpha} k_{t}$ for this case, $H(\alpha) \equiv H(v(\alpha), \alpha)$ takes the form

$$
H(\alpha)=-\left(\frac{(1+\alpha) k_{t}^{2}}{2 c}+\frac{1}{2 n}\right) \cdot v_{U}^{e}(\alpha)^{2}+\frac{\alpha^{2}}{2 n},
$$

where $v_{U}^{e}(\alpha)=\frac{1-\alpha \rho_{U}^{2}(\alpha)}{1+\left(1+\frac{c}{n}\right) \rho_{U}^{2}(\alpha)}$. Since $H(0)<0, H(1)>0$ and $v_{U}^{e}(\alpha)$ is concave and increasing for low values of $\alpha$ but decreasing as $\alpha$ gets larger, there is a unique value $\bar{\alpha}$ so that $H(\bar{\alpha})=0$ and the firm strictly prefers a perfect information for all $\kappa$ if $\alpha \geq \bar{\alpha}$.

Finally, to assure that the agent's manipulation level is positive in equilibrium, it must be that $n>c \cdot\left(1+\alpha / v_{j}^{e}\right)$, where $v_{j}^{e}$ is the optimal incentive rate for information system $j \in\{U, I\}$. The critical value follows from the fact that $a_{t}=\frac{v}{c} E[t(\theta) \mid \sigma]-e^{0}=$ $\left(v \cdot\left(\frac{1}{c}-\frac{1}{n}\right)-\frac{\alpha}{n}\right) \cdot E[t(\theta) \mid \sigma]$. Equivalently, we can express this condition as a lower bound on the optimal incentive rate. In fact, the optimal incentive rate must satisfy that $v_{j}^{e}>\underline{v}=\alpha c /(n-c)$ to assure that $a_{t}>0$ in equilibrium. Since $v_{j}^{e}$ is a function of $\alpha, c, n$, and $\rho_{j}^{2}$, this condition implicitly defines the set of model parameters for which the problem has a meaningful economic solution.

Proof of Proposition 6: At date 2, the agent maximizes his expected utility for given contract parameters $v$ and $w$ anticipating his optimal choices at date 3:

$$
\begin{aligned}
\Psi & =E\left[v \cdot y^{o}(v, \sigma)-w-\sum_{i=r, t} C\left(a_{i}^{o}(v, \sigma)\right)\right] \\
& =E\left[\frac{v^{2}}{2 c} \cdot\left((E[r(\theta) \mid \sigma])^{2}+(E[t(\theta) \mid \sigma])^{2}\right)\right] \\
& =\text { const }+\frac{v^{2}}{2 c} \cdot\left(k_{r}^{2}+k_{t}^{2}\right) \cdot \operatorname{Var}(E[\theta \mid \sigma]) .
\end{aligned}
$$

This expression is monotonically increasing in $\operatorname{Var}(E[\theta \mid \sigma])$. It follows that the agent implements a perfect information system for all incentive rates set by the principal. Considering the agent's optimal choice, the principal maximizes her date 2 objective function $\Gamma(v)$ with respect to $v$ and sets the incentive rate $v=v_{I}$ in Lemma 1 . This 
pair of strategies is a unique Nash equilibrium of the game.

Proof of Proposition 7: In line with the results of Propositions 3 and 3, the solution distinguishes early and late (informed) choice of the control level $e$. With early control, the agent's objective function for a given control effort becomes

$$
\Psi_{E}=\Psi-E[v \cdot e \cdot E[t(\theta) \mid \sigma]]=\Psi-v \cdot e \cdot E[t(\theta)]
$$

where the second term in (27) represent's the expected reduction of the agent's performancebased compensation due to the principal's control. This term is linear in $E[t(\theta) \mid \sigma]$ because $e$ is chosen before $\sigma$ is observed. It follows that the agent strictly prefers a perfect information system for all incentives rates. Since early control does not affect the principal's choice of the optimal incentive rate, the equilibrium is the same as in Proposition 6.

With late control the principal chooses $e$ at date 3 after observing $\sigma$. Considering the optimal control level $e^{o}=(\alpha+v) E[t(\theta) \mid \sigma] / n$ in Lemma 3, the agent's date 2 objective with late control reads as

$$
\begin{aligned}
\Psi_{L} & =\Psi-E\left[\frac{v \cdot(\alpha+v) \cdot E[t(\theta) \mid \sigma]^{2}}{n}\right] \\
& =\text { const }+v \cdot\left(\frac{v}{2 c} \cdot\left(k_{r}^{2}+k_{t}^{2}\right)-\frac{k_{t}^{2}}{n}(v+\alpha)\right) \cdot \operatorname{Var}(E[\theta \mid \sigma]) .
\end{aligned}
$$

It follows that the agent's expected profit is increasing in $\operatorname{Var}(E[\theta \mid \sigma])$ if the term in brackets is positive, or if

$$
v>\frac{2 \alpha}{\frac{n}{c}\left(1+\kappa^{2}\right)-1} \equiv \widehat{v}_{D} .
$$

Thus, the agent strictly prefers a perfect system if $v>\widehat{v}_{D}$ and an uninformative system if $v<\widehat{v}_{D}$. Note first that the agent always chooses a perfect information system if $\alpha=0$ because for this case $\widehat{v}_{D}=0$ so that the agent strictly prefers a perfectly informative system for all incentive rates.

Thus, an equilibrium in which the agent prefers an uninformative system for some parameters requires that $\alpha$ is sufficiently large so that the optimal incentive rate can be lower than $\widehat{v}_{D}$. Let $v(\alpha, \kappa)$ denote the incentive rate as a function of the parameters $\alpha$ and $\kappa$. A sufficient condition for the existence of more than one equilibrium is $\widehat{v}_{D}(\alpha, 0)>$ $v_{U}^{e}(\alpha, 0)$, where $v_{U}^{e}(\alpha, 0)$ is the lowest possible incentive rate for an uninformative system for a given value of $\alpha$ considering that $v_{U}^{e}$ is increasing in $\kappa$. This condition implicitly defines a unique value $\underline{\alpha}>0$ for which the equilibria in Proposition 7 exist.

Suppose that $\alpha>\underline{\alpha}$. Considering the optimal incentive rates for the late control model in Lemma 3, it is easy to see that there are two Nash equilibria in pure strategies and one Nash equilibrium in mixed strategies. Particularly, if $v_{I}^{e} \geq \widehat{v}_{D}$, the agent implements a perfect information system and the principal sets the optimal incentive 
rate $v_{I}^{e}$. It is easy to see that this is the only equilibrium if $\kappa \geq 1$ because in this case, it holds that $v_{I}^{e} \geq v_{U}^{e} \geq \widehat{v}_{D}$ for all incentive rates $v_{j}^{e}>\underline{v}, j \in\{I, U\}$, where $\underline{v}=\alpha c /(n-c)$ is the lowest incentive rate that induce a positive manipulation level in equilibrium as defined in Lemma 3.

In contrast, if $\kappa<1$, there are two more equilibria because $v_{I}^{e}<v_{U}^{e}$. First, if $\widehat{v}_{D}>v_{U}^{e}>v_{I}^{e}$, the agent implements an uninformative system and the principal sets the optimal incentive rate $v_{U}^{e}$. However if $v_{U}^{e} \geq \widehat{v}_{D}>v_{I}^{e}$, there is no pure strategy equilibrium that satisfies the agent's condition for optimal information design and the principal's first-order condition. In this case, there is no equilibrium in pure strategies because if the agent chooses $\operatorname{Var}(E[\theta \mid \sigma])=0$, the principal sets $v=v_{U}^{e}$ but since $v_{U}^{e}>\widehat{v}_{D}$, the agent would like to deviate and choose $\operatorname{Var}(E[\theta \mid \sigma])=\operatorname{Var}(\theta)$. However, if the agent chooses a perfect information system, the principal sets $v=v_{I}^{e}$. Again, since $v_{I}^{e}<\widehat{v}_{D}$, the agent would like to deviate and choose the uninformative system.

The equilibrium in this case is in mixed strategies. Let $\lambda \in[0,1]$ denote the probability the agent assigns to the choice of a perfect information system. The equilibrium solves the following pair of maximization problems

$$
\begin{array}{ll}
\max _{v} & \lambda \cdot \Gamma(v, \operatorname{Var}(\theta))+(1-\lambda) \cdot \Gamma(v, 0), \\
\max _{\lambda} & \lambda \cdot \Psi_{L}(v, \operatorname{Var}(\theta))+(1-\lambda) \cdot \Psi_{L}(v, 0),
\end{array}
$$

where $\Gamma(v, \operatorname{Var}(\theta))$ and $\Psi_{L}(v, \operatorname{Var}(\theta))$ are the principal's and the agent's expected date 2 payoffs for a perfectly informative system and $\Gamma(v, 0)$ and $\Psi_{L}(v, 0)$ are the principal's and the agent's expected date 2 payoffs for an uninformative system, respectively. It is easy to see that the strategy profile

$$
v=\widehat{v}_{D}, \quad \lambda=\frac{\Gamma_{v}\left(\widehat{v}_{D}, 0\right)}{\Gamma_{v}\left(\widehat{v}_{D}, 0\right)-\Gamma_{v}\left(\widehat{v}_{D}, \operatorname{Var}(\theta)\right)}
$$

solves the pair of first-order conditions of the player's maximization problems (28) and (29). Note that $\lambda \in(0,1)$ because we are in the region where $v_{U}>\widehat{v}_{D}>v_{I}$. Specifically, $\Gamma_{v}\left(\widehat{v}_{D}, 0\right)>0$ because $v_{U}>\widehat{v}_{D}$ and $\Gamma_{v}\left(\widehat{v}_{D}, \operatorname{Var}(\theta)\right)<0$ because $\widehat{v}_{D}>v_{I}$. 


\section{Appendix B: Modeling information design}

Option 1. The design of the information system can be portrayed by a function $\chi(\sigma, \theta): \Theta \times \Sigma \rightarrow[0,1]$. It determines the probability with which the feasible signal realizations are produced for each state realization. Given $\chi$, any signal realization $\sigma$ updates the beliefs about $\theta$ to $F_{\sigma ; \chi}(\theta \mid \sigma ; \chi)$ which is equivalent to $G(\theta \mid \sigma)$ in our base model. A perfect information system is characterized by $\chi(\sigma, \theta)=1$ if $\sigma=\theta$. An uninformative system is characterized by $\chi(\sigma, \theta)=$ const for any $\sigma$ and $\theta$.

To illustrate with a specific example, suppose that $\Theta=\left\{\theta_{L}, \theta_{H}\right\}$ and $\Sigma=\left\{\sigma_{L}, \sigma_{H}\right\}$. The prior distribution is represented by $\operatorname{Pr}\left(\theta=\theta_{i}\right)$, whereas the choice of information design is represented by $\chi\left(\theta_{i}, \sigma_{j}\right)=\operatorname{Pr}\left(\sigma_{j} \mid \theta_{i}\right)$ and the conditional distribution by $\operatorname{Pr}\left(\theta_{j} \mid \sigma_{i}\right)$ for all $i, j=H, L$. Consistent beliefs imply that the condition $\operatorname{Pr}\left(\theta_{j}\right)=\operatorname{Pr}\left(\sigma_{i}\right) \operatorname{Pr}\left(\theta_{j} \mid \sigma_{i}\right)+\operatorname{Pr}\left(\sigma_{-i}\right) \operatorname{Pr}\left(\theta_{j} \mid \sigma_{-i}\right)$ has to hold for all $i, j=H, L$. A perfect information system is characterized by $\chi\left(\theta_{i}, \sigma_{i}\right)=\operatorname{Pr}\left(\sigma_{i} \mid \theta_{i}\right)=1$ and an uninformative system by $\chi\left(\theta_{i}, \sigma_{j}\right)=\operatorname{Pr}\left(\sigma_{j} \mid \theta_{i}\right)=1 / 2$, for all $i, j=H, L$.

Option 2. The information system can be portrayed by an additive signal technology

$$
\sigma=\theta+\delta^{-1} \cdot \epsilon
$$

where $\delta$ is a nonnegative parameter and $\epsilon$ is a noise term with mean zero and variance $\operatorname{Var}(\epsilon)$, drawn from a given probability distribution with density $h(\epsilon)$ and unbounded support. Using standard methods for the bivariate transformation of random variables, the joint distribution of $\theta$ and $\sigma$ takes the form

$$
g(\theta, \sigma)=f(\theta) \cdot h(\delta \cdot(\sigma-\theta)) \cdot \delta
$$

The parameter $\delta$ completely characterizes the information system. For finite and positive values of $\delta, g(\theta, \sigma)$ defines the distribution of posterior beliefs $g(\theta \mid \sigma)=\frac{g(\theta, \sigma)}{\int g(\theta, \sigma) d \theta}$, where higher values of $\delta$ reduce the variance of the signal and make the information system more informative. As special cases, $\delta \rightarrow \infty$ represents a perfect information system and $\delta \rightarrow 0$ represents an uninformative system. 


\section{References}

Armstrong, C., A. Jagolinzer, and D. Larcker (2010): Chief Executive Officer Equity Incentives and Accounting Irregularities. Journal of Accounting Research 48 (2), 225-271.

Arya, A., J. Glover, and K. Sivaramakrishnan (1997a): Commitment issues in budgeting. Journal of Accounting Research 35, 273-278.

Arya, A., J. Glover, and K. Sivaramakrishnan (1997b): The Interaction between Decision and Control Problems and the Value of Information. The Accounting Review 72(4), 561-574.

Arya, A., J. Glover, and S. Sunder (1998): Earnings management and the revelation principle. Review of Accounting Studies 3, 7-34.

Baber, W., P. Fairfield, and J. Haggard (1991): The effect of concern about reported income on discretionary spending decisions: the case of research and development. The Accounting Review 66, 818-829.

Baber, W., S. Kang, and L. Liang (2007): Shareholder Rights, Corporate Governance, and Accounting Restatement. Working paper.

Baiman, S. and J. H. Evans (1983): Pre-decision Information and participative management control systems. Journal of Accounting Research 21(2), 371-395.

Baiman, S. and K. Sivaramakrishnan (1991): The value of private pre-decision information in a principal-agent context. The Accounting Review 66(4), 747-766.

Baker, G.P. (1992): Incentive contracts and performance measurement. Journal of Political Economy 100(3), 598-614.

Benston, G. J. and A. L. Hartgraves (2002): Enron: what happened and what we can learn from it. Journal of Accounting and Public Policy 21, 105-127.

Bertomeu, J. and E. Cheynel (2015): Asset measurement in imperfect credit markets. Journal of Accounting Research 53, 965-984.

Beyer, A., I. Guttman, and I. Marinovic (2014): Optimal contracts with performance manipulation, Journal of Accounting Research 52(4), 817-847.

Bhojraj, S., P. Hribar, M. Picconi, and J. McInnis (2009): Making sense of cents: an examination of firms that marginally miss or beat analyst forecasts. Journal of Finance 64 (5), 2361-2388.

Boleslavsky, R. and K. Kim, Bayesian persuasion and moral hazard (March 2018). Available at SSRN: https://ssrn.com/abstract=2913669

Brynjolfsson, E. and K. McElheran (2016): The rapid adoption of data-driven decisionmaking, American Economic Review 106(5), 133-139. 
Bushman, R., R. Indjejikian, and M. Penno (2000): Private pre-decision information, performance measure congruity, and the value of delegation. Contemporary Accounting Research $17(4), 561-87$.

Cagwin, D. and M. J. Bouwman (2002): The association between activity-based costing and improvement in financial performance. Management Accounting Research 13, 1-39.

Caskey, J. and B. Ozel (2017): Earnings expectations and employee safety. Journal of Accounting and Economics 63 (1), 121-141.

Christensen, J. A. and J. Demski (2003): Accounting theory. McGraw-Hill Irwin: Boston.

Christensen, P. O. and G. A. Feltham (2005): Economics of accounting - Volume II, Performance evaluation. Springer: New York.

Crocker, K. J. and J. Slemrod (2007): The economics of earnings manipulation and managerial compensation. The RAND Journal of Economics 38, 698-713.

Cohen, D., R. Mashruwala, and T. Zach (2010): The use of advertising activities to meet earnings benchmarks: evidence from monthly data. Review of Accounting Studies 15 (4), 808-832.

Cohen, D. and P. Zarowin (2010): Accrual-based and real earnings management activities around seasoned equity offerings. Journal of Accounting and Economics 50 (1), 2-19.

Demski, J. (1998): Performance measure manipulation. Contemporary Accounting Research $15,261-85$.

Demski, J., H. Frimor, and D. Sappington (2004): Efficient manipulation in a repeated setting. Journal of Accounting Research 42(1), 31-49.

Dutta, S. and Q. Fan (2014): Equilibrium earnings management and managerial compensation in a multi-period agency setting. Review of Accounting Studies, 1-31.

Dutta, S. and F. Gigler (2002): The effect of earnings forecasts on earnings management. Journal of Accounting Research 40(3), 631-655.

Dye, R. (1988): Earnings management in an overlapping generations model. Journal of Accounting Research 26, 195-235.

Dye, R. and S. Sridhar (2004): Reliability-relevance trade-offs and the efficiency of aggregation. Journal of Accounting Research 42(1), 51-88.

Elson, C. M., C. Ferrere, and N.J. Goossen (2015): The bug at Volkswagen: lessons in codetermination, ownership, and board structure. Journal of Applied Corporate Finance 27(4), $36-43$.

Erickson, M., M. Hanlon, And E. Maydew (2006): Is There a Link between Executive Equity Incentives and Accounting Fraud? Journal of Accounting Research 44, 113-143. 
Ewert, A. and R. Wagenhofer (2005): Economic effects of tightening accounting standards to restrict earnings management. The Accounting Review 80(4), 1101-1124.

Ewert, R. and A. Wagenhofer (2018). Does conservative accounting increase welfare of agencies? The role of endogenous accounting quality. Working paper.

Feltham, G. and J. Xie (1994): Performance measure congruity and diversity in multi-task principal/agent relations. The Accounting Review 69, 429-53.

Fischer, P. and R. Verrecchia (2000): Reporting bias. The Accounting Review 75(2), 229-245.

Friedman, H., J. Hughes, and B. Michaeli (2018): Optimal reporting when additional information might arrive. Working paper.

Gentzkow M. and E. Kamenica (2017): Competition in persuasion. The Review of Economic Studies 84, 300-322.

Goldman, E. and S. Slezak (2006): An equilibrium model of incentive contracts in the presence of information manipulation. Journal of Financial Economics 80, 603-26.

Georgiades G. and B. Szentes (2018): Optimal monitoring design. Working paper.

Göx, R. and A. Wagenhofer (2009): Optimal impairment rules. Journal of Accounting and Economics 48, 2-16.

Harris, J., and P. Bromiley (2007): Incentives to Cheat: The Influence of Executive Compensation and Firm Performance on Financial Misrepresentation. Organizational Science 18, $350 ? 67$.

Hofmann, C. and N. Rothenberg (2013): Interim performance measures and private information. The Accounting Review 88(5), 1683-1714.

Holmström, B. and P. Milgrom (1991): Multi-task principal-agent analyses: incentive contracts, asset ownership, and job design. Journal of Law, Economics and Organization 7, 24-52.

Huang, Z., (2016): Optimal reporting systems with investor information acquisition. Working paper.

Ittner, C., W.N. Lanen and D. Larcker (2002): The association between activity-based costing and manufacturing performance. Journal of Accounting Research 40(3), 711-726.

Kamenica, E. and M. Gentzkow (2011): Bayesian persuasion. American Economic Review 101(6), 2590-2615.

Larcker D., S. Richardson, and I. Tuna (2007): Corporate Governance, Accounting Outcomes, and Organizational Performance. The Accounting Review 82, 963-1008.

Larcker, D. and B. Tayan (2016): Corporate governance matters: a closer look at organizational choices and their consequences, 2nd Edition. Pearson: Old Tappan, NJ. 
Liang, P. J. (2004): Equilibrium earnings management, incentive contracts, and accounting standards. Contemporary Accounting Research 21, 685-718.

Marcec, D. (2018): CEO tenure rates. Harvard Law School Forum on Corporate Governance and Financial Regulation, February 12, 2018.

Michaeli, B. (2017): Divide and inform: rationing information to facilitate persuasion. The Accounting Review 92(5), 167-199.

Pae, S. and S. W. Yoo (2001): Strategic interaction in auditing: an analysis of auditor's legal liability, internal control system quality, and audit effort. The Accounting Review 76(3), 333-356.

Penno, M. (1984): Asymmetry of pre-decision information and managerial accounting. Journal of Accounting Research 22(1), 177-91.

Pozen, R. and S.P. Kothari (2017): Harvard Business Review 95(4), 78-84.

Roychowdhury, S. (2006): Earnings management through real activities manipulation. Journal of Accounting and Economics 42 (3), 335-370.

Rozenbaum, O. (2017): EBITDA and managers' investment and leverage choices. Contemporary Accounting Review 36, 513-546.

Schantl, S. and A. Wagenhofer (2018). Should we regulate firms' internal controls? On the demand for and optimal design of internal control regulation. Working paper.

Sherman H. D. and S. D. Young (2018): The pitfalls of non-GAAP metrics. Sloan Management Review 59 (2), 56-64.

Stein, J. (1989): Efficient capital markets, inefficient firms: a model of myopic corporate behavior. Quarterly Journal of Economics 104(4), 655-669. 